SNUTP 94-20

hep-th/9402117

\title{
A Renormalization Group Flow Approach to Decoupling and Irrelevant Operators
}

\author{
Chanju Kim* \\ Department of Physics and Center for Theoretical Physics, \\ Seoul National University, Seoul, 151-742, Korea
}

\begin{abstract}
Using Wilson-Polchinski renormalization group equations, we give a simple new proof of decoupling in a $\phi^{4}$-type scalar field theory involving two real scalar fields (one is heavy with mass $M$ and the other light). Then, to all orders in perturbation theory, it is shown that effects of virtual heavy particles up to the order $1 / M^{2 N_{0}}$ can be systematically incorporated into light-particle theory via effective local vertices of canonical dimension at most $4+2 N_{0}$. The couplings for vertices of dimension $4+2 N$ are of order $1 / M^{2 N}$ and are systematically calculable. All this is achieved through intuitive dimensional arguments without resorting to complicated graphical arguments or convergence theorems.
\end{abstract}

* e-mail: cjkim@phyb.snu.ac.kr 


\section{INTRODUCTION}

The concept of effective field theories has played an important role in modern theoretical physics and it acquires its natural physical interpretation in the Wilson renormalization group formalism [1]. In the latter, one integrates out the high frequency modes scanned by a cutoff and then considers lowering the cutoff. This generates the RG flow, and the differential equation governing this flow is called the exact renormalization group equation or the flow equation. In general, as we scale down to a lower cutoff, the (Wilsonian) effective Lagrangian converges toward a finite-dimensional manifold parametrized by the "relevant" couplings which correspond to the renormalizable couplings in the conventional perturbation theory. This explains the phenomenological success of renormalizable quantum field theories in particle physics. Polchinski was able to give a simple proof of the perturbative renormalizability within this framework [2], taking the $\phi^{4}$-theory as his model. Since the idea is very general, it has been extended to many different field-theory models, to study renormalization of composite operators and shortdistance expansions, and also toward more practical applications. See for example [3]-10].

If we take seriously the above point of view on effective field theory and renormalization, it should be natural also to find, using this flow equation approach, an intuitive, yet rigorous, proof of the decoupling theorem [1] which states that, in a generic renormalizable quantum field theory with heavy particles of mass $M$, heavy-particle effects decouple from low-energy lightparticle physics except for renormalization of couplings involving light fields and corrections of order $1 / M^{2}$. In fact, as far as low-energy physics is concerned, heavy fields are not very different from massive regulator fields and hence those fields may be integrated out [12] (together with high-frequency modes for light fields) as one lowers the cutoff below the heavy mass scale $M$. One then expects that the nonlocal irrelevant terms, generated in the effective Lagrangian by this integrating-out procedure, be suppressed as the cutoff is scaled further down, assuming that they are well-behaved. In this light, decoupling looks quite natural. Indeed, the decoupling theorem can be proved precisely along this line, and in this approach complicated graphical arguments or convergence theorems are entirely dispensed with.

Since the resulting effective theory is renormalizable to the zeroth order in $1 / M^{2}$, if one wishes to understand the low-energy manifestations of heavy particles, irrelevant (nonrenormalizable) terms must be considered. This issue was investigated in Refs. [13, 14] and the result is that virtual heavy particle effects can be isolated via a set of effective local vertices with calculable couplings when combined with appropriate calculation rules to deal with irrelevant vertices. This factorization theorem was proved to order $1 / M^{2}$ by adapting Zimmermann's algebraic identities [15] in the BPHZ formulation. The proof involves elaborate mathematical rearrangement and convergence theorems, and thus looks a bit artificial. [Also, as for the subgraphs involving heavy and light particle legs simultaneously, the precise nature of factorization is not very clear in this approach.] In this paper, we present a rather elementary proof of this heavy-mass factorization to all orders in perturbation theory and to any given order in $1 / M^{2}$, using flow equations. Actually the whole scheme allows a natural physical interpretation from the viewpoint of the RG flow. The calculation rules for irrelevant vertices are also systematically given. 
There is, however, a subtle point. Our hope is to find a local effective theory with the UV cutoff $\Lambda_{0}(\gg M)$ which is accurate up to the order $1 / M^{2 N_{0}}$ ( $N_{0}$ is any fixed integer). In the original Wilson's view, the cutoff scale of the effective theory may well be identified with the heavy mass scale $M$, above which it is no more effective, and there is no need to worry about the presence of irrelevant terms in the Lagrangian in particular, since "natural scale" of those terms will be around $M$. Among them we may choose to keep explicitly some minimal number of irrelevant terms in our effective Lagrangian! for the accuracy of order $1 / M^{2 N_{0}}$. But, in the conventional discussion of quantum field theory, the UV cutoff $\Lambda_{0}$ is supposed to go to infinity eventually. So, to connect it with Wilson's view, we may suppose scaling up the cutoff of the above Wilsonian effective Lagrangian from $M$ to $\Lambda_{0}$. It will then generate infinitely many irrelevant terms which are unnaturally large. Also, during the scaling, all of them get mixed together and so we are forced to work with the Lagrangian consisting of infinitely many terms all the time. Any kind of truncation for the bare Lagrangian to some finite number of terms would yield divergences in physical quantities as $\Lambda_{0} \rightarrow \infty$, because the unnaturally large coefficients would be amplified by some positive power of $\Lambda_{0} / M$ as the cutoff is scaled down. This is nothing but the statement of nonrenormalizability in the language of RG flow. To avoid this problem we need to deal with irrelevant terms carefully, i.e., give suitable rules to obtain unambiguous finite results with only a finite number of terms included in the bare Lagrangian. It is achieved through the modification of the flow equation when irrelevant vertices are inserted, in the more-or-less same way as one treats composite operators and their normal products [15 in the flow equation approach [5]. Based on this procedure, we can connect the BPHZ approach with the present one.

The plan of our paper is as follows. In Sec. 2, we present some basics on the flow equation, which are used throughout this paper. In Sec. 3, we define our model theory (the full theory) and choose a suitable form of cutoff functions which implement naturally the idea of effective field theory discussed above. We then obtain certain bounds on Green functions which are useful in the next section and prove the perturbative renormalizability. In Sec. 4, we prove the decoupling theorem and then proceed to show the factorization of virtual heavy-particle effects by deriving the local low-energy effective field theory which describes low-energy light-particle physics to any desired order in powers of $1 / M^{2}$. We conclude in Sec. 5. In the Appendix, we briefly describe the renormalization of Green functions with single or multiple insertion of local vertices and Zimmermann's normal product which are needed in Sec. 4.

\section{THE FLOW EQUATION}

In this section we present some features of the flow equation in general setting to fix notations and to facilitate our later analysis. For simplicity we consider a theory of a single

\footnotetext{
${ }^{1}$ For example, the continuum version of Symanzik's improved action [16 in lattice theory have been discussed in 17 by adding suitable irrelevant terms in Lagrangian.
} 
scalar field in four Euclidean dimensions with a cutoff $\Lambda_{0}$. The bare action is written as

$$
S^{\Lambda_{0}}[\phi]=\frac{1}{2}\left\langle\phi, P^{\Lambda_{0}-1} \phi\right\rangle+L^{\Lambda_{0}}[\phi]
$$

where $P^{\Lambda_{0}}$ is the free-particle cutoff propagator, $\langle f, g\rangle$ is defined by the momentum-space integral of $f$ and $g,\langle f, g\rangle \equiv \int \frac{d^{4} p}{(2 \pi)^{4}} f(p) g(-p)$, and $L^{\Lambda_{0}}[\phi]$ represents the interaction part of the bare action. We will also allow the insertions of some additional local vertices or certain composite operators (of unspecified physical origin). To account for this, we define $L_{\text {tot }}^{\Lambda_{0}}[\phi]$ as a formal power series in $\alpha \equiv\left(\alpha_{1}, \ldots, \alpha_{k}\right)$, which has $L^{\Lambda_{0}}[\phi]$ as its zeroth order term, viz.,

$$
L_{\text {tot }}^{\Lambda_{0}}[\phi]=\sum_{|N| \geq 0} \frac{\alpha^{N}}{N !} L^{\Lambda_{0} ; N}[\phi], \quad\left(L^{\Lambda_{0}}[\phi] \equiv L^{\Lambda_{0} ; 0}[\phi]\right)
$$

where $N$ is a multiindex $N \equiv\left(N_{1}, \ldots, N_{k}\right),|N| \equiv \sum_{i=1}^{k} N_{i}, N ! \equiv N_{1} ! \cdots N_{k} !$, and $\alpha^{N} \equiv$ $\alpha_{1}^{N_{1}} \cdots \alpha_{k}^{N_{k}}$. $L^{\Lambda_{0} ; N}$ 's may be regarded as additional local vertices appended to the original Lagrangian $L^{\Lambda_{0}}$ or as composite operators in which one is interested. Also, we will denote

$$
L_{C}^{\Lambda_{0}}[\phi]=\sum_{|N| \geq 1} \frac{\alpha^{N}}{N !} L^{\Lambda_{0} ; N}[\phi] \quad\left(=L_{\text {tot }}^{\Lambda_{0}}[\phi]-L^{\Lambda_{0}}[\phi]\right)
$$

The generating functional, with the insertion of the operator $e^{L_{C}^{\Lambda_{0}}[\phi]}$, is

$$
Z[J]=\int \mathcal{D} \phi e^{-\frac{1}{2}\left\langle\phi, P^{\Lambda_{0}-1} \phi\right\rangle-L_{\text {tot }}^{\Lambda_{0}}[\phi]+\langle J, \phi\rangle} .
$$

Following Wilson and Polchinski [1, 2], one may integrate out the high-momentum components of $\phi$ and reduce the cutoff $\Lambda_{0}$ to a lower scale $\Lambda$. The result is [5, 9]

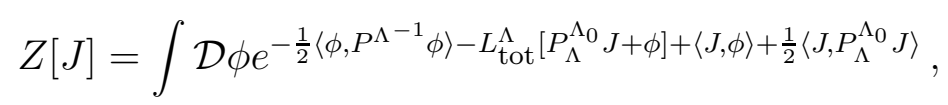

where $P^{\Lambda}\left(P_{\Lambda}^{\Lambda_{0}}\right)$ is the low-(high-)frequency part of the propagatorla with $P^{\Lambda_{0}}=P^{\Lambda}+P_{\Lambda}^{\Lambda_{0}}$, and $L_{\text {tot }}^{\Lambda}$ is the generating functional of amputated connected Green functions (with $e^{L_{C}^{\Lambda_{0}}[\phi]}$ inserted) with both UV cutoff $\Lambda_{0}$ and IR cutoff $\Lambda$. Physical Green functions are obtained from $L_{\text {tot }}^{\Lambda}$ in the limit $\Lambda \rightarrow 0$ [5, 6, 9]. On the other hand, if $J(p)=0$ for $p>\Lambda$ so that $J$ couples to the low-frequency modes only, all $J$ 's drop out from Eq. (2.5) except for $\langle J, \phi\rangle$ since $P_{\Lambda}^{\Lambda_{0}}$ has only high-frequency modes, and $L_{\text {tot }}^{\Lambda}$ coincides with the Wilsonian effective action [1]. $L_{\text {tot }}^{\Lambda}$ obeys the exact renormalization group equation or the flow equation [2],

$$
\partial_{\Lambda} L_{\text {tot }}^{\Lambda}[\phi]=-\left.\frac{1}{2} \int \frac{d^{4} p}{(2 \pi)^{4}} \partial_{\Lambda} P^{\Lambda}(p)\left[\frac{\delta^{2} L_{\mathrm{tot}}^{\Lambda}}{\delta \phi(p) \delta \phi(-p)}-\frac{\delta L_{\mathrm{tot}}^{\Lambda}}{\delta \phi(p)} \frac{\delta L_{\mathrm{tot}}^{\Lambda}}{\delta \phi(-p)}\right]\right|_{\text {field-dep. part }} .
$$

\footnotetext{
${ }^{2}$ There will be infinite ways in doing the separation; choosing one way of separation may be considered as choosing a "renormalization scheme" in the Wilson renormalization group approach, and physical quantities are independent of such choices. While it is not necessary to specify a particular scheme at this stage, we will choose a specific scheme later to facilitate our discussions.
} 
To fix appropriate boundary conditions for $L_{\text {tot }}^{\Lambda}$, renormalization conditions in the conventional renormalization procedure may be used at $\Lambda=0$ where physical Green functions are generated.

Expanding $L_{\text {tot }}^{\Lambda}$ in powers of $\alpha$, we now define $L^{\Lambda ; N}$ 's as the expansion coefficients, i.e.,

$$
L_{\mathrm{tot}}^{\Lambda}[\phi]=\sum_{|N| \geq 0} \frac{\alpha^{N}}{N !} L^{\Lambda ; N}[\phi], \quad\left(L^{\Lambda ; 0}[\phi] \equiv L^{\Lambda}[\phi]\right) .
$$

Then, $L^{\Lambda ; N}[\phi]$ is the generating functional of amputated connected Green functions with an insertion of an operator $O_{N}$ which is identified as the $N$-th order coefficient of $e^{L_{C}^{\Lambda_{0}}[\phi]}$, i.e.,

$$
\sum_{|N| \geq 1} \frac{\alpha^{N}}{N !} O_{N} \equiv e^{L_{C}^{\Lambda_{0}}}=\exp \left(\sum_{|N| \geq 1} \frac{\alpha^{N}}{N !} L^{\Lambda_{0} ; N}\right) .
$$

Explictly, $O_{N}$ can be calculated as

$$
O_{N}=\sum_{\sum_{I_{l}} I_{l} n_{I}=N_{l}} N ! \prod_{|I| \geq 1} \frac{1}{n_{I} !}\left(\frac{L^{\Lambda_{0} ; I}}{I !}\right)^{n_{I}}
$$

where $I=\left(I_{1}, \ldots, I_{k}\right)$ and $I !=I_{1} ! \cdots I_{k}$ !. For example, if $k=1, O_{1}=L^{\Lambda_{0} ; 1}, O_{2}=L^{\Lambda_{0} ; 2}+$ $\left(L^{\Lambda_{0} ; 1}\right)^{2}, O_{3}=L^{\Lambda_{0} ; 3}+3 L^{\Lambda_{0} ; 2} L^{\Lambda_{0} ; 1}+\left(L^{\Lambda_{0} ; 1}\right)^{3}$ and so on. At the zeroth order of $\alpha$, we obtain the flow equation for the effective Lagrangian $L^{\Lambda}$ from Eq. (2.6), which assumes an identical form as Eq. (2.6) other than the replacement $L_{\text {tot }}^{\Lambda} \rightarrow L^{\Lambda}$. At order $\alpha^{N}$, on the other hand, we have

$$
\begin{array}{r}
\partial_{\Lambda} L^{\Lambda ; N}=-\frac{1}{2} \int \frac{d^{4} p}{(2 \pi)^{4}} \partial_{\Lambda} P^{\Lambda}(p)\left[\frac{\delta^{2} L^{\Lambda ; N}}{\delta \phi(p) \delta \phi(-p)}-2 \frac{\delta L^{\Lambda}}{\delta \phi(p)} \frac{\delta L^{\Lambda ; N}}{\delta \phi(-p)}\right. \\
\left.-\sum_{0<I<N}\left(\begin{array}{c}
N \\
I
\end{array}\right) \frac{\delta L^{\Lambda ; I}}{\delta \phi(p)} \frac{\delta L^{\Lambda ; N-I}}{\delta \phi(-p)}\right]\left.\right|_{\text {field-dep. part }}
\end{array}
$$

where $\left(\begin{array}{c}N \\ I\end{array}\right) \equiv\left(\begin{array}{c}N_{1} \\ I_{1}\end{array}\right) \cdots\left(\begin{array}{c}N_{k} \\ I_{k}\end{array}\right)$ and $I \leq N$ means $I_{i} \leq N_{i}$ (for every $\left.i=1, \ldots, k\right)$ while $I<N$ represents $I \leq N$ and $|I|<|N|$. If $k=|N|=1$, i.e., for a single insertion of operator through $L^{\Lambda_{0} ; 1}$, the last term vanishes and so we have a linear and homogeneous equation in $L^{\Lambda ; 1}$, which has been used in discussing composite operators [4, 5]. Equation (2.10) with $k=2$ and $N=(1,1)$ has also appeared in the literature [5] in deriving the short-distance expansion of two composite operators. Note that, if $|N|>1$, Eq. (2.10) contains inhomogeneous terms. However, in spite of the inhomogeneous piece, it is still of first order in $L^{\Lambda ; N}$ while the homogeneous part remains exactly the same for all $N$. Therefore the general solution will be a sum of a particular solution and a solution to the homogeneous equation (which is just the $|N|=1$ equation for Green functions with a single insertion of an operator). This property will be used in the Appendix where Zimmermann's normal product and multiple insertion of local vertices are discussed in this formalism. Equation (2.10) is the starting point of our subsequent analyses. 


\section{STRUCTURES OF THE FULL THEORY}

\subsection{Defining the full theory}

As our full theory we consider a scalar theory ( $\phi$ - $\psi$ theory) which involves two real scalar fields $\phi$ and $\psi$, defined with an ultraviolet cutoff $\Lambda_{0}$. The mass $M$ of $\psi$ field is assumed to be much larger than the mass $m$ of $\phi$ field but of course much smaller than $\Lambda_{0}$. In the next section, we will discuss the effect of the heavy field $\psi$ at low energy. The bare action for the system reads

$$
S^{(f) \Lambda_{0}}=\frac{1}{2}\left\langle\phi, P_{m}^{\Lambda_{0}-1} \phi\right\rangle+\frac{1}{2}\left\langle\psi, P_{M}^{\Lambda_{0}-1} \psi\right\rangle+L^{(f) \Lambda_{0}},
$$

where $P_{m}^{\Lambda_{0}}$ and $P_{M}^{\Lambda_{0}}$ are respective free-particle cutoff propagators. We assume that particles interact via quartic couplings, observing $Z_{2} \times Z_{2}$ symmetry. Explicitly, we may write

$$
L^{(f) \Lambda_{0}}=\int d^{4} x\left[\frac{\rho_{1}^{f}}{2} \phi^{2}+\frac{\rho_{2}^{f}}{2}\left(\partial_{\mu} \phi\right)^{2}+\frac{\rho_{3}^{f}}{4 !} \phi^{4}+\frac{\rho_{4}^{f}}{2} \psi^{2}+\frac{\rho_{5}^{f}}{2}\left(\partial_{\mu} \psi\right)^{2}+\frac{\rho_{6}^{f}}{4 !} \psi^{4}+\frac{\rho_{7}^{f}}{4} \phi^{2} \psi^{2}\right] .
$$

The superscript $f$ is used for quantities of the full theory. As is conventional, the bare couplings $\rho_{a}^{f}(a=1,2, \cdots, 7)$ may be written as

$$
\begin{aligned}
& \rho_{1}^{f}=Z_{\phi} m_{0}^{2}-m^{2}, \quad \rho_{2}^{f}=Z_{\phi}-1, \quad \rho_{3}^{f}=Z_{\phi}^{2} g_{1}^{0}, \\
& \rho_{4}^{f}=Z_{\psi} M_{0}^{2}-M^{2}, \quad \rho_{5}^{f}=Z_{\psi}-1, \quad \rho_{6}^{f}=Z_{\psi}^{2} g_{2}^{0}, \\
& \rho_{7}^{f}=Z_{\phi} Z_{\psi} g_{3}^{0},
\end{aligned}
$$

where $\left(m_{0}^{2}, M_{0}^{2}\right),\left(Z_{\phi}, Z_{\psi}\right)$ and $\left(g_{1}^{0}, g_{2}^{0}, g_{3}^{0}\right)$ are bare masses, wave-function renormalizations and bare coupling constants, respectively. Propagators $P_{m}^{\Lambda_{0}}$ and $P_{M}^{\Lambda_{0}}$ are defined by

$$
P_{m}^{\Lambda_{0}}=\frac{R_{m}\left(\Lambda_{0}, p\right)}{p^{2}+m^{2}}, \quad P_{M}^{\Lambda_{0}}=\frac{R_{M}\left(\Lambda_{0}, p\right)}{p^{2}+M^{2}}
$$

where $R_{m}(\Lambda, p)$ and $R_{M}(\Lambda, p)$ are smoothed variants of the sharp cutoff function $\theta(\Lambda-p)$. As indicated in Sec. 2, choosing $R_{m}$ and $R_{M}$ corresponds to choosing a "renormalization scheme" and physical quantities are not affected. Here we choose the following "mass-dependent scheme":

$$
\begin{aligned}
& R_{m}(\Lambda, p)=\left[1-K\left((1+\Lambda / m)^{2}\right)\right] K\left(\frac{p^{2}}{\Lambda^{2}}\right) \\
& R_{M}(\Lambda, p)=\left[1-K\left(\Lambda^{2} / M^{2}\right)\right] K\left(\frac{p^{2}}{\Lambda^{2}}\right)
\end{aligned}
$$

where $K$ is a $C^{\infty}$-function on $[0, \infty)$ such that $K(a)=1,0 \leq a \leq 1$ and $K(a)=0, a \geq 4$ with $K(a) \rightarrow 1(0)$ exponentially as $a \rightarrow 1(4)$. Thus, both $R_{m}(\Lambda, p)$ and $R_{M}(\Lambda, p)$ are $C^{\infty}$-functions on $\left([0, \infty) \times \mathbb{R}^{4}\right)$ and $R_{m}\left(R_{M}\right)$ approaches 0 exponentially as $\Lambda \rightarrow 0$ (as $\left.\Lambda \rightarrow M\right)$. If $\Lambda>m$ for $R_{m}$ or $\Lambda>2 M$ for $R_{M}$, they simply reduce to $K\left(p^{2} / \Lambda^{2}\right)$. A notable property of $R_{M}$ is that if 
$\Lambda<M$, we have $R_{M}=0$ identically. This choice of $R_{M}$ is very natural for our purpose, because it implies that at the scale $\Lambda=M$ we have all modes of the heavy field $\psi$ integrated out and there remains only the light field $\phi$ below the scale $M$; it explicitly implements Wilson's point of view on effective field theory. Therefore, if $\Lambda<M$, the flow equation will look like that for the theory having the light field only. One may regard this property specific to our choice (3.5) as the analogy of the so-called "manifest decoupling" in the conventional approach [18]. On the contrary, if one chose a "mass-independent scheme", i.e. if $R_{m}$ and $R_{M}$ were chosen independently of their masses $m$ and $M$, a substantial part of heavy particle modes would not be integrated out even below the scale $M$, thus making subsequent discussions rather complicated.

Now, we define $L^{(f) \Lambda}[\phi, \psi]$ following the general line discussed in Sec. 2. It will then satisfy

$\partial_{\Lambda} L^{(f) \Lambda}=-\left.\frac{1}{2} \int \frac{d^{4} p}{(2 \pi)^{4}}\left\{\partial_{\Lambda} P_{m}^{\Lambda}(p)\left[\frac{\delta^{2} L^{(f) \Lambda}}{\delta \phi(p) \delta \phi(-p)}-\frac{\delta L^{(f) \Lambda}}{\delta \phi(p)} \frac{\delta L^{(f) \Lambda}}{\delta \phi(-p)}\right]+(m \rightarrow M, \phi \rightarrow \psi)\right\}\right|_{\text {field-dep. part }}$.

We expand $L^{(f) \Lambda}$ in powers of $\phi$ and $\psi$ in momentum space, utilizing $Z_{2} \times Z_{2}$ symmetry,

$$
\begin{aligned}
L^{(f) \Lambda}[\phi, \psi]=\sum_{|r| \geq 0} \sum_{|n| \geq 1} g^{r} \int \prod_{j=1}^{2 n_{1}} \frac{d^{4} p_{j}}{(2 \pi)^{4}} \prod_{j=1}^{2 n_{2}-1} \frac{d^{4} p_{j}^{\prime}}{(2 \pi)^{4}} \phi\left(p_{1}\right) \cdots \phi\left(p_{2 n_{1}}\right) \psi\left(p_{1}^{\prime}\right) \cdots \psi\left(p_{2 n_{2}-1}^{\prime}\right) \\
\times \mathcal{L}_{r, 2 n}^{(f) \Lambda}\left(p_{1}, \ldots, p_{2 n_{1}} ; p_{1}^{\prime}, \ldots, p_{2 n_{2}-1}^{\prime}\right),
\end{aligned}
$$

where $p_{2 n_{2}}^{\prime} \equiv-\sum_{j=1}^{2 n_{1}} p_{j}-\sum_{j=1}^{2 n_{2}-1} p_{j}^{\prime}$. Some explanations on our notations are in order: $g \equiv$ $\left(g_{1}, g_{2}, g_{3}\right)$ are perturbation-expansion parameters which may be identified as renormalized coupling constants (see below), $r$ and $n$ represent $\left(r_{1}, r_{2}, r_{3}\right)$ and $\left(n_{1}, n_{2}\right)$, respectively, with $|n| \equiv n_{1}+n_{2},|r| \equiv r_{1}+r_{2}+r_{3}$ and finally, $g^{r} \equiv g_{1}^{r_{1}} g_{2}^{r_{2}} g_{3}^{r_{3}}$. Then the vertex function $\mathcal{L}_{r, 2 n}^{(f) \Lambda}$ has Bose symmetry and, in addition, will possess following properties: (i) as we noted in Sec. 2, $\mathcal{L}_{2,2 n}^{(f) \Lambda=0}$ can be identified with amputated connected Green functions $G_{r, 2 n}^{(f) c}$ of the theory, i.e.,

$$
\mathcal{L}_{r, 2 n}^{(f) \Lambda=0}\left(p_{1}, \ldots, p_{2 n_{1}} ; p_{1}^{\prime}, \ldots, p_{2 n_{2}-1}^{\prime}\right)=G_{r, 2 n}^{(f) c}\left(p_{1}, \ldots, p_{2 n_{1}} ; p_{1}^{\prime}, \ldots, p_{2 n_{2}-1}^{\prime}\right) ;
$$

(ii) $\mathcal{L}_{r, 2 n}^{(f) \Lambda}=0$ if $|n|>|r|+1$ since just the connected diagrams contribute to $\mathcal{L}_{r, 2 n}^{(f) \Lambda}$ as we have seen in Sec. 2; (iii) $\mathcal{L}_{r, 2 n}^{(f) \Lambda} \in C^{\infty}\left(\left[0, \Lambda_{0}\right] \times \mathbb{R}^{4(n-1)}\right)$ and is invariant under the $O(4)$ rotation group. For later use, we also denote vertex functions obtained by summing over $r_{2}$ and $r_{3}$ as $\overline{\mathcal{L}}$, i.e.,

$$
\overline{\mathcal{L}}_{r_{1}, 2 n}^{(f) \Lambda} \equiv \sum_{r_{2}, r_{3}} g_{2}^{r_{2}} g_{3}^{r_{3}} \mathcal{L}_{\left(r_{1}, r_{2}, r_{3}\right), 2 n}^{(f) \Lambda} .
$$

We insert the expansion (3.7) into the flow equation (3.6) and arrive at the equation

$$
\begin{aligned}
& \partial_{\Lambda} \mathcal{L}_{r, 2 n}^{(f) \Lambda}\left(p_{1}, \ldots, p_{2 n_{1}} ; p_{1}^{\prime}, \ldots, p_{2 n_{2}-1}^{\prime}\right) \\
& \quad=-\left(\begin{array}{c}
2 n_{1}+2 \\
2
\end{array}\right) \int \frac{d^{4} q}{(2 \pi)^{4}} \partial_{\Lambda} P_{m}^{\Lambda}(q) \mathcal{L}_{r, 2\left(n_{1}+1, n_{2}\right)}^{(f) \Lambda}\left(p_{1}, \ldots, p_{2 n_{1}}, q,-q ; p_{1}^{\prime}, \ldots, p_{2 n_{2}-1}^{\prime}\right)
\end{aligned}
$$




$$
\begin{aligned}
&+2 \sum_{\substack{r^{\prime}+\prime^{\prime \prime}=r \\
l_{1}+l_{1}^{\prime}=n_{1}+1 \\
l_{2}+l_{2}^{\prime}=n_{2}}} l_{1} l_{1}^{\prime} \partial_{\Lambda} P_{m}^{\Lambda}(P) {\left[\mathcal{L}_{r^{\prime}, 2\left(l_{1}, l_{2}\right)}^{(f) \Lambda}\left(p_{1}, \ldots, p_{2 l_{1}-1}, P ; p_{1}^{\prime}, \ldots, p_{2 l_{2}-1}^{\prime}\right)\right.} \\
&-\left(\begin{array}{c}
2 n_{2}+2 \\
2
\end{array}\right) \int \frac{d^{4} q}{(2 \pi)^{4}} \partial_{\Lambda} P_{M}^{\Lambda}(q) \mathcal{L}_{r, 2\left(n_{1}, n_{2}+1\right)}^{(f) \Lambda}\left(p_{1}, \ldots, p_{2 n_{1}} ; p_{1}^{\prime}, \ldots, p_{2 n_{2}}^{\prime}, q\right) \\
&+2 \sum_{\substack{r^{\prime \prime}, 2\left(l_{1}^{\prime}, l_{2}^{\prime}\right) \\
r_{1}^{\prime}+r^{\prime \prime}=r \\
l_{1}+l_{1}^{\prime}=n_{1} \\
l_{2}+l_{2}^{\prime}=n_{2}+1}}^{(f) \Lambda} l_{2} l_{2}^{\prime} \partial_{\Lambda} P_{M}^{\Lambda}\left(P^{\prime}\right)\left[\mathcal{L}_{r^{\prime}, 2\left(l_{1}, l_{2}\right)}^{(f) \Lambda}\left(p_{1}, \ldots, p_{2 n_{1}}, P ; p_{2 l_{2}+1}^{\prime}, \ldots, p_{1}^{\prime}, \ldots, p_{2 l_{2}-1}^{\prime}\right)\right. \\
& \\
&\left.\quad \times \mathcal{L}_{r^{\prime \prime}, 2\left(l_{1}^{\prime}, l_{2}^{\prime}\right)}^{(f) \Lambda}\left(p_{2 l_{1}+1}^{\prime}, \ldots, p_{2 n_{1}} ; p_{2 l_{2}}^{\prime}, \ldots, p_{2 n_{2}}^{\prime}\right)\right]_{s y m m},
\end{aligned}
$$

where $P=-\sum_{j=1}^{2 l_{1}-1} p_{j}-\sum_{j=1}^{2 l_{2}} p_{j}^{\prime}, P^{\prime}=-\sum_{j=1}^{2 l_{1}} p_{j}-\sum_{j=1}^{2 l_{2}-1} p_{j}^{\prime}$ and $[\cdots]_{s y m m}$ implies symmetrization with respect to momenta $p_{1}, \ldots, p_{2 n_{1}}$ (and momenta $p_{1}^{\prime}, \ldots, p_{2 n_{2}}^{\prime}$ ). Denoting $\phi(\psi)$-legs by thin(thick)-lines and $\partial_{\Lambda} P^{\Lambda}$ by a straight line, we can represent this equation by the diagram shown in Fig. 1. Here some remarks on Eq. (3.10) are in order. First, since we have $\mathcal{L}_{0,2 n}^{(f) \Lambda}=0$ (see below) the restriction $r^{\prime}+r^{\prime \prime}=r$ also implies that $r^{\prime}, r^{\prime \prime}<r$ (i.e., $\left|r^{\prime}\right|,\left|r^{\prime \prime}\right|<|r|$ and $\left.r_{i}^{\prime}, r_{i}^{\prime \prime} \leq r_{i}\right)$. Also, in relation with the effective theory of the next section, it should be noted that, for $\mathcal{L}_{r, 2\left(n_{1}, 0\right)}^{(f) \Lambda}$ (i.e., no external heavy particles), the last term is identically zero and $\psi$ enters the flow equation only through the $\Lambda$-differentiated propagator in the third term.

If suitable boundary conditions are given, the flow equation (3.10) will produce the amputated connected Green functions of the theory, $G_{r, 2 n}^{(f) c}=\mathcal{L}_{r, 2 n}^{(f) \Lambda=0}$. From Eq. (3.2), we have

$$
\begin{array}{lll}
\Lambda=\Lambda_{0}: & \partial_{p}^{w} \mathcal{L}_{r, 2 n}^{(f) \Lambda_{0}}=0, & 2|n|+|w|>4 \\
& \partial_{p}^{w} \mathcal{L}_{r, 2 n}^{(f) \Lambda_{0}}(0) \sim \text { bare couplings in Eq. (3.3), } & 2|n|+|w| \leq 4,
\end{array}
$$

(to be determined)

where $w$ is the multiindex $\{w\} \equiv\left\{w_{1}, \ldots, w_{2 n_{1}}, w_{1}^{\prime}, \ldots, w_{2 n_{2}-1}^{\prime}\right\}, w_{j}=\left(w_{j 1}, \ldots, w_{j 4}\right)$ and $\partial_{p}^{w} \equiv$ $\partial_{p_{1}}^{w_{1}} \cdots \partial_{p_{2 n_{1}}}^{w_{2 n_{1}}} \partial_{p_{1}^{\prime}}^{w_{1}^{\prime}} \cdots \partial_{p_{2 n_{1}}^{\prime}}^{w_{2 n_{2}}^{\prime}}, \partial_{p_{i}}^{w_{i}} \equiv \prod_{\mu=1}^{4} \partial^{w_{i \mu}} /\left(\partial p_{i \mu}\right)^{w_{i \mu}}$. We will also write $|w| \equiv \sum_{i, \mu}\left(w_{i \mu}+w_{i \mu}^{\prime}\right)$. At $\Lambda=0$, on the other hand, we impose following renormalization conditions on relevant terms [:

$$
\begin{array}{lll}
\mathcal{L}_{r,(2,0)}^{(f) \Lambda=0}(0)=0, & \partial_{p_{\mu}} \partial_{p_{\nu}} \mathcal{L}_{r,(2,0)}^{(f) \Lambda=0}(0)=0, & \mathcal{L}_{r,(4,0)}^{(f) \Lambda=0}(0)=\frac{1}{4 !} \delta_{r,(1,0,0)}, \\
\mathcal{L}_{r,(0,2)}^{(f) \Lambda=0}\left(\bar{p}_{1}^{\prime}\right)=0, & \partial_{p_{\mu}} \partial_{p_{\nu}} \mathcal{L}_{r,(0,2)}^{(f) \Lambda=0}\left(\bar{p}_{1}^{\prime}\right)=0, & \mathcal{L}_{r,(0,4)}^{(f) \Lambda=0}\left(\bar{p}_{2}^{\prime}, \bar{p}_{3}^{\prime}, \bar{p}_{4}^{\prime}\right)=\frac{1}{4 !} \delta_{r,(0,1,0)}, \\
\mathcal{L}_{r,(2,2)}^{(f) \Lambda=0}\left(0, \bar{p}_{5}^{\prime}\right)=\frac{1}{4} \delta_{r,(0,0,1) .} &
\end{array}
$$

Here, normalization momenta $\bar{p}_{i}^{\prime}(i=1, \ldots, 5)$ are chosen to be constants of magnitude $M$; i.e., we have chosen the renormalization points for the light-particle Green functions at zero

\footnotetext{
${ }^{3}$ With these conditions the expansion parameters $\left(g_{1}, g_{2}, g_{3}\right)$ are now identified with renormalized coupling constants. As far as our proof goes, details of the renormalization conditions are not important as long as one choose the renormalization points for the light particle at momenta much smaller than $M$ [18]. But, for practical applications, it would be necessary to choose the renormalization conditions for the heavy particles at momentum values of order the heavy particle mass 19$]$.
} 
momentum, and those for the heavy particles at momenta of magnitude $M$. It is then easy to show that $\rho_{a}^{f}$ are uniquely fixed within perturbation theory. (See, for example, Ref. [5].)

\subsection{Bounds on Green functions}

Now the theory being completely specified, one may use the flow equation to demonstrate the perturbative renormalizability [2, 5], which amounts to boundedness and convergence of the vertex functions $\mathcal{L}_{r, 2 n}^{(f) \Lambda}$ as $\Lambda_{0} \rightarrow \infty$. To reach our goal in the next section, however, we need slightly stronger bounds which take particle-mass dependence into account explicitly.

First, we introduce a set of norms on vertex functions \|\|$_{\left(a, b, b^{\prime}\right)}$, for positive real $a, b$, and $b^{\prime}$,

$$
\left\|\partial^{z} \mathcal{L}_{r, 2\left(n_{1}, n_{2}\right)}^{(f) \Lambda}\right\|_{\left(a, b, b^{\prime}\right)} \equiv \max _{\substack{\left|p_{i}\right| \leq \max \{a, b\} \\\left|p_{i}^{\prime}\right| \leq \max \left\{a, b^{\prime}\right\} \\|w|=z}}\left|\partial_{p}^{w} \mathcal{L}_{r, 2\left(n_{1}, n_{2}\right)}^{(f) \Lambda}\left(p_{1}, \ldots, p_{2 n_{1}} ; p_{1}^{\prime}, \ldots, p_{2 n_{2}-1}^{\prime}\right)\right| .
$$

For vertices with no heavy-particle leg, we will often omit $b^{\prime},\left\|\partial^{z} \mathcal{L}_{r, 2\left(n_{1}, 0\right)}^{(f) \Lambda}\right\|_{(a, b)} \equiv\left\|\partial^{z} \mathcal{L}_{r, 2\left(n_{1}, 0\right)}^{(f) \Lambda}\right\|_{\left(a, b, b^{\prime}\right)}$. Now it is not difficult to show that, with the choice (3.5), we have the following uniform bounds:

$$
\left|\frac{\partial_{p}^{z} \partial_{\Lambda} R_{m}(\Lambda, p)}{\left(p^{2}+m^{2}\right)^{n}}\right| \leq \frac{c}{(\Lambda+m)^{2 n+z+1}}, \quad\left|\frac{\partial_{p}^{z} \partial_{\Lambda} R_{M}(\Lambda, p)}{\left(p^{2}+M^{2}\right)^{n}}\right| \leq \frac{c^{\prime}}{\Lambda^{2 n+z+1}} \theta(\Lambda-M),
$$

where $0 \leq \Lambda \leq \Lambda_{0}$ and $c, c^{\prime}$ are constants which do not depend on $\Lambda, \Lambda_{0}, M$, or $m$ (but may depend on $z, n$ and $k$ ). Acting $\partial_{p}^{w}$ on Eq. (3.10) and estimating the resulting expression with the help of the bounds (3.14), we find that, for a fixed constant $\mu$ of order $m$,

$$
\begin{aligned}
& \left\|\partial_{\Lambda} \partial^{z} \mathcal{L}_{r, 2 n}^{(f) \Lambda}\right\|_{(2 \Lambda, \mu, M)} \\
& \leq \operatorname{const}\left\{(\Lambda+m)\left[\left\|\partial^{z} \mathcal{L}_{r, 2\left(n_{1}+1, n_{2}\right)}^{(f) \Lambda}\right\|_{(2 \Lambda, \mu, M)}+\theta(\Lambda-M)\left\|\partial^{z} \mathcal{L}_{r, 2\left(n_{1}, n_{2}+1\right)}^{(f) \Lambda}\right\|_{(2 \Lambda, \mu, M)}\right]\right. \\
& \left.+\left(\sum_{\substack{r^{\prime}+r^{\prime \prime}=r \\
l+l^{\prime}=\left(n_{1}+1, n_{2}\right) \\
z_{1}+z_{2}+z_{3}=z}}+\theta(\Lambda-M) \sum_{\substack{r^{\prime}+r^{\prime \prime}=r \\
l+l^{\prime}=\left(n_{1}, n_{2}+1\right) \\
z_{1}+z_{2}+z_{3}=z}}\right) \frac{1}{(\Lambda+m)^{3+z_{1}}}\left\|\partial^{z_{2}} \mathcal{L}_{r^{\prime}, 2\left(l_{1}, l_{2}\right)}^{(f) \Lambda}\right\|_{(2 \Lambda, \mu, M)}\left\|\partial^{z_{3}} \mathcal{L}_{r^{\prime \prime}, 2\left(l_{1}^{\prime}, l_{2}^{\prime}\right)}^{(f) \Lambda}\right\|_{(2 \Lambda, \mu, M)}\right\},
\end{aligned}
$$

where "const" stands for some finite number independent of $M, \Lambda$ and $\Lambda_{0}$ (but may depend on $m$ and $\mu$ through $(\mu / m))$. Equation (3.15) holds for all $\Lambda \in\left[0, \Lambda_{0}\right]$, and it allows us to present our subsequent results and proofs in simpler forms than those appeared in earlier works [2, 5].

Now we are ready to prove the boundeness of vertex functions:

Theorem 1 For vertices with no external heavy-particle leg,

$$
\left\|\partial^{z} \mathcal{L}_{r, 2\left(n_{1}, 0\right)}^{(f) \Lambda}\right\|_{(2 \Lambda, \mu, M)} \leq(\Lambda+m)^{4-2 n_{1}-z} \operatorname{Plog}\left(\frac{\Lambda+m}{m}\right), \quad 0 \leq \Lambda \leq \Lambda_{0},
$$

while, for irrelevant vertices $(2|n|+z>4)$ with external heavy-particle legs,

$$
\left\|\partial^{z} \mathcal{L}_{r, 2 n}^{(f) \Lambda}\right\|_{(2 \Lambda, \mu, M)} \leq(\Lambda+m)^{4-2|n|-z} \mathrm{P} \log \left(\frac{\Lambda+M}{m}\right), \quad 0 \leq \Lambda \leq \Lambda_{0}
$$


and, finally, for relevant vertices $(2|n|+4 \leq 4)$ with external heavy-particle legs,

$$
\left\|\partial^{z} \mathcal{L}_{r, 2 n}^{(f) \Lambda}\right\|_{(2 \Lambda, \mu, M)} \leq(\Lambda+M)^{4-2|n|-z} \operatorname{Plog}\left(\frac{\Lambda+M}{m}\right), \quad 0 \leq \Lambda \leq \Lambda_{0}
$$

where $\mathrm{P} \log (\cdot)$ is some polynomial in $\log (\cdot)$ whose coefficients are independent of $M, \Lambda$, and $\Lambda_{0}$.

Before proving Theorem 1, we here note that the above bounds are not unexpected — they just show the right scaling behaviors. That is, the effect of imposing renormalization conditions at momenta of order $M$ for heavy-particle legs shows with appropriate powers of $M$ (up to logarithmic corrections) in the flow equation of vertices with external heavy-particle legs. On the other hand, in the light-particle sector, the bounds have the same form as those of a single scalar theory [2, 5]: the large $M$ corrections do not show up because $m$ is forced to be small by hand. Of course, this should be the case for decoupling to occur in the first place.

Proof of Theorem 1. We proceed along the induction scheme set up in Ref. [2]. Equations (3.16) -(3.18) are trivially true for $r=0$ or for $(r, n)$ with $|r|>0$ and $|n|>|r|+1$, because $\mathcal{L}_{r, 2 n}^{(f) \Lambda}=0$ for those $(r, n)$-values as we have seen before. Now suppose it holds for $(r, n)$ satisfying the condition $|r| \leq s-1$, or $|r|=s$ and $|n| \geq k+1$. Then the induction step consists in proving Eqs. (3.16)-(3.18) for any fixed $(r, n)$ with $|r|=s$ and $|n|=k$. We begin with the irrelevant vertices, i.e., with the case $2 k+z>4$. For these we use the boundary condition (3.11) to write

$$
\left\|\partial^{z} \mathcal{L}_{r, 2 n}^{(f) \Lambda}\right\|_{(2 \Lambda, \mu, M)} \leq \int_{\Lambda}^{\Lambda_{0}} d \lambda\left\|\partial_{\lambda} \partial^{z} \mathcal{L}_{r, 2 n}^{(f) \lambda}\right\|_{(2 \Lambda, \mu, M)}
$$

The right hand side is now estimated with the help of Eq. (3.15). If $\Lambda>M$, according to the induction hypothesis it is easy to find that, for irrelevant vertices with no heavy-particle leg(f,

$$
\begin{aligned}
\left\|\partial^{z} \mathcal{L}_{r, 2\left(n_{1}, 0\right)}^{(f) \Lambda}\right\|_{(2 \Lambda, \mu, M)} & \leq \int_{\Lambda}^{\Lambda_{0}} d \lambda(\lambda+m)^{3-2 k-z} \operatorname{Plog}\left(\frac{\lambda}{m}\right) \\
& \leq(\Lambda+m)^{4-2 k-z} \operatorname{Plog}\left(\frac{\Lambda+m}{m}\right) .
\end{aligned}
$$

A similar estimate yields the bound (3.17) for irrelevant vertices with heavy-particle legs. For $\Lambda \leq M$, the bounds on $\partial_{p}^{w} \mathcal{L}_{r,(0,2)}^{(f) \Lambda}$ with $z=0,1$ in Eq. (3.18) look problematic because they are not suppressed by appropriate powers of $\Lambda$, i.e., they might result in undesirable extra powers of $M$ when one estimates the right hand side of Eq. (3.15) with the induction hypothesis. They, however, cause no real problem since they appear in the right hand side of Eq. (3.15) only with $\theta(\Lambda-M)$. There are other dangerous terms when one estimates vertices with $n_{2}=0$ for $\Lambda<M$, namely, the $\log M / m$ corrections in Eqs. (3.17) and (3.18); they are again multiplied by $\theta(\Lambda-M)$ and do not contribute at all if $\Lambda<M$. Therefore, we conclude that the induction argument used in $\Lambda>M$ case goes through with no obstuction for irrelevant vertices.

\footnotetext{
${ }^{4}$ Each time the symbol Plog appears, it stands in general for a new polynomial of log.
} 
For the case of relevant vertices $(2 k+z \leq 4)$, we begin with $\mathcal{L}_{r,(0,4)}^{(f) \Lambda}$. Recalling the boundary conditions at $\Lambda=0$, we integrate the flow equation (3.10) from 0 to $\Lambda$ (at $p_{i}^{\prime}=\bar{p}_{i}^{\prime}$ ) to obtain

$$
\begin{aligned}
\left|\mathcal{L}_{r,(0,4)}^{(f) \Lambda}\left(\bar{p}_{i}^{\prime}\right)\right| & \leq\left|\mathcal{L}_{r,(0,4)}^{(f) \Lambda=0}\left(\bar{p}_{i}^{\prime}\right)\right|+\int_{0}^{\Lambda} d \lambda\left\|\partial_{\lambda} \mathcal{L}_{r,(0,4)}^{(f) \lambda}\right\|_{(2 \Lambda, \mu, M)} \\
& \leq \operatorname{Plog}\left(\frac{\Lambda+M}{m}\right)
\end{aligned}
$$

where we have used the induction hypothesis in the second line. Now, using the Taylor's formula

$$
\mathcal{L}_{r,(0,4)}^{(f) \Lambda}\left(p_{i}^{\prime}\right)=\mathcal{L}_{r,(0,4)}^{(f) \Lambda}\left(\bar{p}_{i}^{\prime}\right)+\left.\sum_{i=1}^{3}\left(p_{i}^{\prime}-\bar{p}_{i}^{\prime}\right) \int_{0}^{1} d t \partial_{q_{i \mu}} \mathcal{L}_{r,(0,4)}^{(f) \Lambda}\left(q_{i}\right)\right|_{q_{i}=t p_{i}},
$$

and then putting $\Lambda=M$, we get a bound for $\mathcal{L}_{r,(0,4)}^{(f) \Lambda=M}$ as being of order $\operatorname{Plog}(M / m)$ at most. Then the integration of $\partial_{\lambda} \mathcal{L}_{r,(0,4)}^{(f) \lambda}$ from $\Lambda$ to $M$ gives Eq. (3.18). The same argument works for other relevant vertices with $2 k+z=4$. Next, if $n=(0,1)$ and $z=1$, we may use the property that $\partial_{p}^{w} \mathcal{L}_{r, 2 n}^{(f) \Lambda}(0)=0$ for odd dimensional vertices (due to $Z_{2} \times Z_{2}$ symmetry), and therefore

$$
\begin{aligned}
\left\|\partial \mathcal{L}_{r,(0,2)}^{(f) \Lambda}\right\|_{(2 \Lambda, \mu, M)} & =\left\|\left.p_{\mu}^{\prime} \int_{0}^{1} d t \partial_{q_{\mu}^{\prime}} \partial_{q^{\prime}} \mathcal{L}_{r,(0,2)}^{(f) \Lambda}\left(q^{\prime}\right)\right|_{q^{\prime}=t p^{\prime}}\right\|_{(2 \Lambda, \mu, M)} \\
& \leq(\Lambda+M) \operatorname{Plog}\left(\frac{\Lambda+M}{m}\right) .
\end{aligned}
$$

The same argument shows that $\left\|\partial \mathcal{L}_{r,(2,0)}^{(f) \Lambda}\right\|_{(2 \Lambda, \mu, M)} \leq(\Lambda+m) \operatorname{Plog}\left(\frac{\Lambda+m}{m}\right)$. It is then straightforward to obtain the bounds for $|n|=1$ and $z=0$ using Taylor expansion around the renormalization point, as we did for $2 k+z=4$ case.

In particular, at $\Lambda=0$, Theorem 1 tells us that amputated connected Green functions and their derivatives of the theory are bounded, for $\left|p_{i}\right| \leq \mu$ and $\left|p_{i}^{\prime}\right| \leq M$, by

$$
\left|\partial_{p}^{w} G_{r, 2 n}^{(f) c}\left(p_{1}, \ldots, p_{2 n_{1}} ; p_{1}^{\prime}, \ldots, p_{2 n_{2}-1}^{\prime}\right)\right| \leq \begin{cases}\operatorname{const} \cdot m^{4-2 n_{1}-|w|}, & n_{2}=0 \\ m^{4-2|n|-|w|} \operatorname{Plog}\left(\frac{M}{m}\right), & n_{2} \neq 0,2|n|+|w|>4 \\ M^{4-2|n|-|w|} \operatorname{Plog}\left(\frac{M}{m}\right), & n_{2} \neq 0,2|n|+|w| \leq 4 .\end{cases}
$$

Now we show that Green functions converges to a finite limit as the UV cutoff $\Lambda_{0}$ goes to infinity. It will also complete the proof of the perturbative renormalizability of the $\phi-\psi$ theory.

Theorem 2 For vertices with no external heavy-particle leg,

$$
\left\|\partial_{\Lambda_{0}} \partial^{z} \mathcal{L}_{r, 2\left(n_{1}, 0\right)}^{(f) \Lambda}\right\|_{(2 \Lambda, \mu, M)} \leq\left(\frac{\Lambda+m}{\Lambda_{0}}\right)^{3}(\Lambda+m)^{3-2 n_{1}-z} \operatorname{Plog}\left(\frac{\Lambda_{0}}{m}\right), \quad 0 \leq \Lambda \leq \Lambda_{0},
$$

while, for irrelevant vertices $(2|n|+z>4)$ with external heavy-particle legs,

$$
\left\|\partial_{\Lambda_{0}} \partial^{z} \mathcal{L}_{r, 2 n}^{(f) \Lambda}\right\|_{(2 \Lambda, \mu, M)} \leq\left(\frac{\Lambda+M}{\Lambda_{0}}\right)^{2}\left(\frac{\Lambda+m}{\Lambda_{0}}\right)(\Lambda+m)^{3-2|n|-z} \operatorname{Plog}\left(\frac{\Lambda_{0}}{m}\right), \quad 0 \leq \Lambda \leq \Lambda_{0}
$$


and, finally, for relevant vertices $(2|n|+4 \leq 4)$ with external heavy-particle legs,

$$
\left\|\partial_{\Lambda_{0}} \partial^{z} \mathcal{L}_{r, 2 n}^{(f) \Lambda}\right\|_{(2 \Lambda, \mu, M)} \leq\left(\frac{\Lambda+M}{\Lambda_{0}}\right)^{3}(\Lambda+M)^{3-2|n|-z} \operatorname{Plog}\left(\frac{\Lambda_{0}}{m}\right), \quad 0 \leq \Lambda \leq \Lambda_{0} .
$$

Proof. The method is very similar to that employed in Theorem 1 and we only sketch the procedure. For irrelevant vertices, we use the identity

$$
\partial_{\Lambda_{0}} \partial_{p}^{w}\left(\mathcal{L}_{r, 2 n}^{(f) \Lambda_{0}}-\mathcal{L}_{r, 2 n}^{(f) \Lambda}\right)=\left.\partial_{p}^{w} \partial_{\Lambda} \mathcal{L}_{r, 2 n}^{(f) \Lambda}\right|_{\Lambda=\Lambda_{0}}+\int_{\Lambda}^{\Lambda_{0}} d \lambda \partial_{\lambda} \partial_{\Lambda_{0}} \partial_{p}^{w} \mathcal{L}_{r, 2 n}^{(f) \lambda}
$$

while, for relevant vertices, we use the relation

$$
\partial_{\Lambda_{0}} \partial_{p}^{w}\left(\mathcal{L}_{r, 2 n}^{(f) \Lambda}-\mathcal{L}_{r, 2 n}^{(f) \Lambda=0}\right)=\int_{0}^{\Lambda} d \lambda \partial_{\lambda} \partial_{\Lambda_{0}} \partial_{p}^{w} \mathcal{L}_{r, 2 n}^{(f) \lambda}
$$

Then, differentiating Eq. (3.10) with respect to $\Lambda_{0}$, we obtain the bounds for the right hand sides, as in Eq. (3.15). Finally, applying the induction scheme as described in Theorem 1 and using the results of Theorem 1, it is straightforward to prove the theorem.

\section{DECOUPLING, FACTORIZATION AND EFFECTIVE LAGRANGIANS}

We are now ready to discuss the low-energy physics of the $\phi-\psi$ theory. In this section we will prove the decoupling theorem and the factorization of virtual heavy-particle effects to any desired order in $1 / M^{2}$ on the basis of the flow equation approach. We start with proving the decoupling theorem which corresponds to the zeroth order result of factorization.

\subsection{Low-energy effective field theory and the decoupling theorem}

Let us consider a theory of light particles (the $\phi$ theory) of mass $m$ with the interaction part $L^{\Lambda_{0}}$ of the bare action given by

$$
L^{\Lambda_{0}}=\int d^{4} x\left[\frac{1}{2} \rho_{1} \phi^{2}(x)+\frac{1}{2} \rho_{2}\left(\partial_{\mu} \phi(x)\right)^{2}+\frac{1}{4 !} \rho_{3} \phi^{4}(x)\right] .
$$

As before, $L^{\Lambda}[\phi]$ is defined to satisfy the flow equation (2.6) and can be expanded as

$$
L^{\Lambda}[\phi]=\sum_{r=0}^{\infty} \sum_{n=1}^{\infty} g_{1}^{r} \int_{j=1}^{2 n-1} \prod_{j}^{4} \frac{d_{j}}{(2 \pi)^{4}} \phi\left(p_{1}\right) \cdots \phi\left(p_{2 n}\right) \mathcal{L}_{r, 2 n}^{\Lambda}\left(p_{1}, \ldots, p_{2 n-1}\right)
$$

The vertex function $\mathcal{L}_{r, 2 n}^{\Lambda}$ has properties similar to those for $\mathcal{L}_{r, 2 n}^{(f) \Lambda}: \mathcal{L}_{r, 2 n}^{\Lambda}$ has Bose symmetry, $\mathcal{L}_{r, 2 n}^{\Lambda}=0$ if $n>r+1, \mathcal{L}_{r, 2 n}^{\Lambda}$ is $C^{\infty}$ and Euclidean-invariant, and finally $\mathcal{L}_{r, 2 n}^{\Lambda=0}$ is equal to the 
amputated connected Green function of the theory, i.e., $G_{r, 2 n}^{c}$. It satisfies the flow equation

$$
\begin{aligned}
\partial_{\Lambda} \mathcal{L}_{r, 2 n}^{\Lambda}\left(p_{1}, \ldots, p_{2 n-1}\right) & =-\left(\begin{array}{c}
2 n+2 \\
2
\end{array}\right) \int \frac{d^{4} q}{(2 \pi)^{4}} \partial_{\Lambda} P_{m}^{\Lambda}(q) \mathcal{L}_{r, 2(n+1)}^{\Lambda}\left(q,-q, p_{1}, \ldots, p_{2 n-1}\right) \\
& +2 \sum_{\substack{r^{\prime}+r^{\prime \prime}=r \\
l+l^{\prime}=n+1}} l l^{\prime} \partial_{\Lambda} P_{m}^{\Lambda}(P)\left[\mathcal{L}_{r^{\prime}, 2 l}^{\Lambda}\left(p_{1}, \ldots, p_{2 l-1}\right) \mathcal{L}_{r^{\prime \prime}, 2 l^{\prime}}^{\Lambda}\left(-P, p_{2 l}, \ldots, p_{2 n-1}\right)\right]_{s y m m},
\end{aligned}
$$

where $P=-\sum_{j=1}^{2 n-1} p_{j}$. The boundary conditions which $\mathcal{L}_{r, 2 n}^{\Lambda}$ 's obey are determined as follows. First, at $\Lambda=\Lambda_{0}$, irrelevant vertices vanish, i.e.,

$$
\partial_{p}^{w} \mathcal{L}_{r, 2 n}^{\Lambda_{0}}=0, \quad 2 n+|w|>4
$$

At $\Lambda=0$, we impose the same renormalization conditions (3.12) as those in the full theory,

$$
\mathcal{L}_{r, 2}^{\Lambda=0}(0)=\partial_{p_{\mu}} \partial_{p_{\nu}} \mathcal{L}_{r, 2}^{\Lambda=0}(0)=0, \quad \mathcal{L}_{r, 4}^{\Lambda=0}(0)=\frac{1}{4 !} \delta_{r 1},
$$

so that the $\phi$ theory may not be distinguishable from the $\phi$ - $\psi$ theory at low energies.

From Theorems 11 and 2 we immediately have the bounds on the vertex functions $\mathcal{L}_{r, 2 n}^{\Lambda}$ (which amounts to proving the perturbative renormalizabililty of the $\phi$ theory), viz.,

$$
\begin{aligned}
& \left\|\partial^{z} \mathcal{L}_{r, 2 n}^{\Lambda}\right\|_{(2 \Lambda, \mu)} \leq(\Lambda+m)^{4-2 n-z} \operatorname{Plog}\left(\frac{\Lambda+m}{m}\right), \quad 0 \leq \Lambda \leq \Lambda_{0}, \\
& \left\|\partial_{\Lambda_{0}} \partial^{z} \mathcal{L}_{r, 2 n}^{\Lambda}\right\|_{(2 \Lambda, \mu)} \leq\left(\frac{\Lambda+m}{\Lambda_{0}}\right)^{3}(\Lambda+m)^{3-2 n-z} \operatorname{Plog}\left(\frac{\Lambda_{0}}{m}\right), \quad 0 \leq \Lambda \leq \Lambda_{0} .
\end{aligned}
$$

Now we are in a position to prove the decoupling theorem:

Theorem 3 The difference of the vertex functions $\mathcal{L}_{r, 2(n, 0)}^{(f) \Lambda}$ in the $\phi$ - $\psi$ theory and $\mathcal{L}_{r_{1}, 2 n}^{\Lambda}$ in the $\phi$ theory, where $r=\left(r_{1}, r_{2}, r_{3}\right)$, satisfies the bound

$$
\left\|\partial^{z}\left(\mathcal{L}_{r, 2(n, 0)}^{(f) \Lambda}-\mathcal{L}_{r_{1}, 2 n}^{\Lambda} \delta_{r,\left(r_{1}, 0,0\right)}\right)\right\|_{(2 \Lambda, \mu)} \leq \begin{cases}\left(\frac{\Lambda+m}{M}\right)^{2}(\Lambda+m)^{4-2 n-z} \operatorname{Plog}\left(\frac{M}{m}\right), & 0 \leq \Lambda \leq M \\ \Lambda^{4-2 n-z} \operatorname{Plog}\left(\frac{\Lambda}{m}\right) . & M \leq \Lambda \leq \Lambda_{0},\end{cases}
$$

Proof. If $\Lambda \in\left[M, \Lambda_{0}\right]$, the bound is immediate from Eqs. (3.16) and (4.6), and hence it suffices to consider $0 \leq \Lambda \leq M$ only. Let us define $\mathcal{D}_{r, 2 n}^{\Lambda}$ as the difference of vertex functions,

$$
\mathcal{D}_{r, 2 n}^{\Lambda} \equiv \mathcal{L}_{r, 2(n, 0)}^{(f) \Lambda}-\mathcal{L}_{r_{1}, 2 n}^{\Lambda} \delta_{r,\left(r_{1}, 0,0\right)}
$$

Subtracting the flow equation (3.10) for $\mathcal{L}_{r, 2 n}^{(f) \Lambda}$ from that for $\mathcal{L}_{r, 2 n}^{\Lambda}$ (given in (4.3)) and making estimates using Eq. (3.14), one easily finds that, for $0 \leq \Lambda \leq M$,

$$
\begin{aligned}
& \left\|\partial_{\Lambda} \partial^{z} \mathcal{D}_{r, 2 n}^{\Lambda}\right\|_{(2 \Lambda, \mu)} \leq \operatorname{const}\left\{(\Lambda+m)\left\|\partial^{z} \mathcal{D}_{r, 2(n+1)}^{\Lambda}\right\|_{(2 \Lambda, \mu)}\right. \\
& \left.\quad+\sum_{\substack{r^{\prime}+r^{\prime \prime}=r \\
l+l^{\prime}=n+1 \\
z_{1}+z_{2}+z_{3}=z}} \frac{1}{(\Lambda+m)^{3+z_{1}}}\left\|\partial^{z_{2}} \mathcal{D}_{r^{\prime}, 2 l}^{\Lambda}\right\|_{(2 \Lambda, \mu)}\left(\left\|\partial^{z_{3}} \mathcal{D}_{r^{\prime \prime}, 2 l^{\prime}}^{\Lambda}\right\|_{(2 \Lambda, \mu)}+\left\|\partial^{z_{3}} \mathcal{L}_{r_{1}^{\prime \prime}, 2 l^{\prime}}^{\Lambda}\right\|_{(2 \Lambda, \mu)} \delta_{r^{\prime \prime},\left(r_{1}^{\prime \prime}, 0,0\right)}\right)\right\}
\end{aligned}
$$


where it should be noted that terms involving the heavy-particle propagator all vanish. Boundary conditions for $\mathcal{D}_{r, 2 n}^{\Lambda}$ are simply

$$
\begin{aligned}
& \partial_{p}^{w} \mathcal{D}_{r, 2 n}^{\Lambda_{0}}=0, \quad \text { for } 2 n+|w|>4 \\
& \mathcal{D}_{r, 2}^{\Lambda=0}(0)=\partial_{p_{\mu}} \partial_{p_{\nu}} \mathcal{D}_{r, 2}^{\Lambda=0}(0)=\mathcal{D}_{r, 4}^{\Lambda=0}(0)=0 .
\end{aligned}
$$

Since $\mathcal{D}_{r, 2 n}^{\Lambda}=0$ if $r=0$ or $n>|r|+1$ with $|r|>0$, the theorem holds trivially for these values of $r, n$. So we will now prove the validity of Eq. (4.8) assuming that it is true for $r$ and $n$ satisfying $|r| \leq s-1$ or $|r|=s, n \geq k+1$. Pick any particular $r$ satisfying $|r|=s$. As usual we first consider the case of irrelevant vertices. For $2 k+z>5$, we may use the same argument leading to Eq. (3.20) in the proof of Theorem 1 and find that

$$
\begin{aligned}
\left\|\partial^{z} \mathcal{D}_{r, 2 k}^{\Lambda}\right\|_{(2 \Lambda, \mu)} & \leq\left\|\partial^{z} \mathcal{D}_{r, 2 k}^{M}\right\|_{(2 \Lambda, \mu)}+\int_{\Lambda}^{M} d \lambda\left\|\partial_{\lambda} \partial^{z} \mathcal{D}_{r, 2 k}^{\Lambda}\right\|_{(2 \lambda, \mu)} \\
& \leq M^{4-2 k-z} \operatorname{Plog}\left(\frac{M}{m}\right)+\int_{\Lambda}^{M} d \lambda \frac{(\lambda+m)^{5-2 k-z}}{M^{2}} \operatorname{Plog}\left(\frac{M}{m}\right) \\
& \leq \frac{(\Lambda+m)^{6-2 k-z}}{M^{2}} \operatorname{Plog}\left(\frac{M}{m}\right),
\end{aligned}
$$

where, in the second line, we used the induction hypothesis. If $2 k+z=5$, we may use the property that $\partial_{p}^{w} \mathcal{D}_{r, 2 k}^{\Lambda}(0)=0$ for odd dimensional vertices and therefore

$$
\left\|\partial^{z} \mathcal{D}_{r, 2 k}^{\Lambda}\right\|_{(2 \Lambda, \mu)}=\left\|\left.\int_{0}^{1} d t \sum_{i=1}^{2 n-1} p_{i \mu} \partial_{q_{i \mu}} \partial_{q}^{w} \mathcal{D}_{r, 2 k}^{\Lambda}\left(q_{1}, \ldots, q_{2 n-1}\right)\right|_{q_{i}=t p_{i}}\right\|_{(2 \Lambda, \mu)}
$$

to obtain the bound (4.8). The case with relevant vertices (i.e., with $2 k+z \leq 4$ ) is quite trivial. As in the proof of Theorem 1, we use the boundary conditions (4.11) at $\Lambda=0$ and obtain

$$
\left|\partial_{p}^{w} \mathcal{D}_{r, 2 k}^{\Lambda}(0)\right| \leq \int_{0}^{\Lambda} d \lambda\left\|\partial_{\lambda} \partial^{z} \mathcal{D}_{r, 2 k}^{\lambda}\right\|_{(2 \lambda, \mu)} .
$$

Then Taylor's formula (3.22), with $\mathcal{L}_{r, 2 n}^{(f) \Lambda}$ replaced by $\mathcal{D}_{r, 2 n}^{\Lambda}$ and also using the argument immediately after Eq. (3.22), yields the desired bound.

Therefore, the difference between amputated connected Green functions of the $\phi$ - $\psi$ theory and those of the $\phi$ theory is bounded by $\left(\frac{m}{M}\right)^{2} m^{4-2 n-z} \operatorname{Plog}\left(\frac{M}{m}\right)$ for $\left|p_{i}\right| \leq \mu$, which is the standard statement of the decoupling theorem. Also note that the difference in the vertex functions is no longer small if $\Lambda$ becomes comparable to the heavy particle mass $M$, which implies that low-energy effective theory is not useful above the heavy particle mass scale.

In fact, as we discussed in Sec. 1, the decoupling theorem may look more or less obvious if one takes the view that only the light field is subject to direct observation. One may then imagine a pure $\phi$-field theory with the Lagrangian given from the Lagrangian of the $\phi-\psi$ theory after integrating out the heavy field, and identify the cutoff $\Lambda_{0}$ with the heavy particle mass scale $M$. What we have shown above is then that (i) integration of the heavy field and the "identification" of the cutoff $\Lambda_{0}$ with the heavy particle mass scale can be achieved by our "mass-dependent scheme", Eq. (3.5), and (ii) the resulting nonlocal irrelevant terms are indeed well-behaved so that decoupling theorem may follow. 


\subsection{Factorization of virtual heavy particle effects}

We now examine higher order terms in $1 / M^{2}$, which originate from virtual heavy-particle interactions. Let us suppose that we want to describe low-energy physics in the full theory accurately up to order $1 / M^{2 N_{0}}$. ( $N_{0}$ is some positive integer.) We will show below that we can then factorize all heavy-particle effects to the given order by making use of Eq. (2.10) (with $k=1$ ) and by appropriately choosing the operators $L^{\Lambda_{0} ; N}, N=1,2, \ldots, N_{0}$.

Here, $L^{\Lambda_{0} ; N}$ 's may be assumed to have the form

$$
L^{\Lambda_{0} ; N}=\int d^{4} x\left(\begin{array}{c}
\text { local, Euclidean invariant, even polynomials } \\
\text { in } \phi \text { and its derivatives, of dim. } \leq 4+2 N
\end{array}\right), \quad N=1,2, \ldots, N_{0} .
$$

The coefficient of the polynomials will be chosen later so that $L^{\Lambda_{0} ; N}$ carries information appropriate to $O\left(1 / M^{2 N}\right)$-effects from the full theory. Then the sum of $L^{\Lambda_{0} ; N}$ 's, viz.,

$$
\widetilde{L}^{\Lambda_{0} ; N_{0}} \equiv L^{\Lambda_{0}}+\sum_{N=1}^{N_{0}} \frac{1}{N !} L^{\Lambda_{0} ; N}\left(=\widetilde{L}^{\Lambda_{0} ; N_{0}-1}+\frac{1}{N_{0} !} L^{\Lambda_{0} ; N_{0}}\right),
$$

will reproduce the predictions of the full theory at low energy with an accuracy of order $1 / M^{2 N_{0}}$. The quantity $L^{\Lambda ; N}$, which satisfies the flow equation (2.10), may be expanded in powers of $\phi$ :

$$
L^{\Lambda ; N}[\phi]=\sum_{r=0}^{\infty} \sum_{n=1}^{\infty} g_{1}^{r} \int \prod_{j=1}^{2 n-1} \frac{d^{4} p_{j}}{(2 \pi)^{4}} \phi\left(p_{1}\right) \cdots \phi\left(p_{2 n}\right) \mathcal{L}_{r, 2 n}^{\Lambda ; N}\left(p_{1}, \ldots, p_{2 n-1}\right)
$$

We may also define $\widetilde{\mathcal{L}}_{r, 2 n}^{\Lambda ; N}$, s through the same kind of relation. Then the vertex function $\mathcal{L}_{r, 2 n}^{\Lambda ; N}$ is $C^{\infty}$ and satisfies the properties similar to those of $\mathcal{L}_{r, 2 n}^{(f) \Lambda}$; in particular, there is a finite number $n_{0}(r, N)$ such that $\mathcal{L}_{r, 2 n}^{\Lambda ; N}=0$ if $n>n_{0}(r, N) . \mathcal{L}_{r, 2 n}^{\Lambda ; N}$ 's obey the flow equation

$$
\begin{aligned}
\partial_{\Lambda} \mathcal{L}_{r, 2 n}^{\Lambda ; N}\left(p_{1}, \ldots, p_{2 n-1}\right) \\
\quad=-\left(\begin{array}{c}
2 n+2 \\
2
\end{array}\right) \int \frac{d^{4} q}{(2 \pi)^{4}} \partial_{\Lambda} P_{m}^{\Lambda}(q) \mathcal{L}_{r, 2(n+1)}^{\Lambda ; N}\left(q,-q, p_{1}, \ldots, p_{2 n-1}\right) \\
\quad+2 \sum_{I=0}^{N} \sum_{\substack{r^{\prime}+r^{\prime \prime}=r \\
l+l^{\prime}=n+1}} l l^{\prime} \partial_{\Lambda} P_{m}^{\Lambda}(P)\left(\begin{array}{c}
N \\
I
\end{array}\right)\left[\mathcal{L}_{r^{\prime}, 2 l}^{\Lambda ; I}\left(p_{1}, \ldots, p_{2 l-1}\right) \mathcal{L}_{r^{\prime \prime}, 2 l^{\prime}}^{\Lambda ; N-I}\left(-P, p_{2 l}, \ldots, p_{2 n-1}\right)\right]_{s y m m} \\
\quad \equiv \mathcal{F}_{r, 2 n}^{\Lambda ; N}\left(p_{1}, \ldots, p_{2 n-1}\right)
\end{aligned}
$$

where $P=-\sum_{j=1}^{2 l-1} p_{j}$. To complete the definition of $L^{\Lambda_{0} ; N}$, we must also state the boundary conditions for $\mathcal{L}_{r, 2 n}^{\Lambda ; N}$. At $\Lambda=\Lambda_{0}$, Eq. (4.15) implies that

$$
\partial_{p}^{w} \mathcal{L}_{r, 2 n}^{\Lambda_{0} ; N}=0, \quad \text { for } 2 n+|w|>4+2 N
$$

For $2 n+|w| \leq 4+2 N$, we impose the following conditions, recursively in $N$ :

$$
\partial_{p}^{w} \mathcal{L}_{r, 2 n}^{\Lambda=0 ; N}(0)=N ! \partial_{p}^{w}\left(\overline{\mathcal{L}}_{r, 2 n}^{(f) \Lambda=0}(0)-\widetilde{\mathcal{L}}_{r, 2 n}^{\Lambda=0 ; N-1}(0)\right)
$$


where $\overline{\mathcal{L}}$ is defined in Eq. (3.9). (Consequently, $\partial_{p}^{w} \mathcal{L}_{r, 2 n}^{\Lambda=0 ; N}(0)=0$ for the case $2 n+|w|<4+2 N$.) It remains to show that the boundary conditions (4.19) and (4.20) define $L^{\Lambda_{0} ; N}$ 's uniquely. For this, we use Eq. (4.18) together with the conditions (4.19) and (4.20) to rewrite

$$
\mathcal{L}_{r, 2 n}^{\Lambda_{0} ; N}=\tau^{4+2 N-2 n} \mathcal{L}_{r, 2 n}^{\Lambda=0 ; N}+\int_{0}^{\Lambda_{0}} d \lambda \tau^{4+2 N-2 n} \mathcal{F}_{r, 2 n}^{\lambda ; N}
$$

where the symbol $\tau^{l}$ stands for the Taylor operator defined by

$$
\left.\tau^{l} f\left(p_{1}, \ldots, p_{n}\right) \equiv \sum_{j=0}^{l} \frac{1}{j !}\left(\frac{d}{d t}\right)^{j} f\left(t p_{1}, \ldots, t p_{n}\right)\right|_{t=0} \quad(\text { for } l \geq 0)
$$

(and $\tau^{l} f \equiv 0$ for $l<0$ ). Then using induction on $N, r$, and $n$ one may easily confirm that $L^{\Lambda_{0} ; N}$ 's are defined uniquely.

The theory being completely specified, we will now prove the factorization. To begin with, we define $\mathcal{D}_{r, 2 n}^{\Lambda ; N}$, where $r$ is a multiindex $r=\left(r_{1}, r_{2}, r_{3}\right)$ from now on, as

$$
\mathcal{D}_{r, 2 n}^{\Lambda ; N} \equiv \mathcal{L}_{r, 2(n, 0)}^{(f) \Lambda}-{\widetilde{\mathcal{L}^{\prime}}}_{r, 2 n}^{\Lambda ; N} \quad\left(=\mathcal{D}_{r, 2 n}^{\Lambda ; N-1}-\mathcal{L}_{r, 2 n}^{\prime \Lambda ; N} / N !\right)
$$

where $\mathcal{L}_{r, 2 n}^{\prime \Lambda ; N}$ (and $\widetilde{\mathcal{L}_{r, 2 n}^{\prime}}{ }_{r ; N}^{\Lambda ; N}$, correspondingly) is defined by the expansion, $\mathcal{L}_{r_{1}, 2 n}^{\Lambda ; N}=\sum_{r_{2}, r_{3}} g_{2}^{r_{2}} g_{3}^{r_{3}} \mathcal{L}_{\left(r_{1}, r_{2}, r_{3}\right), 2 n}^{\Lambda}$. The flow equation satisfied by $\mathcal{D}_{r, 2 n}^{\Lambda ; N}$ will then be the difference between those given in Eqs. (3.10) and (4.18). After some algebra, we find that the equation can be written in the form

$$
\begin{aligned}
\partial_{\Lambda} \mathcal{D}_{r, 2 n}^{\Lambda ; N}= & -\left(\begin{array}{c}
2 n+2 \\
2
\end{array}\right) \int \frac{d^{4} q}{(2 \pi)^{4}}\left[\partial_{\Lambda} P_{m}^{\Lambda} \mathcal{D}_{r, 2(n+1)}^{\Lambda ; N}+\partial_{\Lambda} P_{M}^{\Lambda} \mathcal{L}_{r,(2 n, 2)}^{(f) \Lambda}\right] \\
& +2 \sum_{\substack{r^{\prime}+r^{\prime \prime}=r \\
l+l^{\prime \prime}=n+1}} l l^{\prime} \partial_{\Lambda} P_{m}^{\Lambda}\left[\sum_{I=1}^{N} \frac{1}{I !} \mathcal{L}_{r^{\prime}, 2 l}^{\Lambda ; I} \mathcal{D}_{r^{\prime \prime}, 2 l^{\prime}}^{\Lambda ; N-I}+2 \mathcal{L}_{r^{\prime}, 2 l}^{\Lambda \Lambda} \mathcal{D}_{r^{\prime \prime}, 2 l^{\prime}}^{\Lambda ; N}+\mathcal{D}_{r^{\prime}, 2 l}^{\Lambda} \mathcal{D}_{r^{\prime \prime}, 2 l^{\prime}}^{\Lambda ; N}\right]_{s y m m},
\end{aligned}
$$

where, for simplicity, we have omitted the arguments of vertex functions which are the same as those in Eq. (3.10) or (4.18). The boundary conditions satisfied by $\mathcal{D}_{r, 2 n}^{\Lambda ; N}$ are very simple:

$$
\begin{array}{rll}
\text { at } \Lambda=\Lambda_{0}: & \partial_{p}^{w} \mathcal{D}_{r, 2 n}^{\Lambda_{0} ; N}=0, & \text { for } 2 n+|w|>4+2 N, \\
\text { at } \Lambda=0: & \partial_{p}^{w} \mathcal{D}_{r, 2 n}^{\Lambda=0 ; N}(0)=0, & \text { for } 2 n+|w| \leq 4+2 N .
\end{array}
$$

Note that if it were not for the inhomogeneous term containing $\mathcal{L}_{r, 2 n}^{(f) \Lambda}$ in Eq. (4.24), our boundary condition (4.25) would imply that $\mathcal{D}_{r, 2 n}^{\Lambda ; N}$ should vanish identically. In other words, a nonzero result for the difference between the vertex functions of the $\phi-\psi$ theory and those of the $\phi$ theory is due to the inhomogeneous term which is in fact nonzero only for large $\Lambda$, i.e., for $\Lambda \geq M$. As was done in previous sections, we can obtain an estimate for $\mathcal{D}_{r, 2 n}^{\Lambda ; N}$ :

$$
\left\|\partial_{\Lambda} \partial^{z} \mathcal{D}_{r, 2 n}^{\Lambda ; N}\right\|_{(2 \Lambda, \mu)} \leq \operatorname{const}\left\{(\Lambda+m)\left\|\partial^{z} \mathcal{D}_{r, 2(n+1)}^{\Lambda ; N}\right\|_{(2 \Lambda, \mu)}+\theta(\Lambda-M) \Lambda\left\|\partial^{z} \mathcal{L}_{r,(2 n, 2)}^{(f) \Lambda}\right\|_{(2 \Lambda, \mu, M)}\right.
$$




$$
\begin{gathered}
+\sum_{\substack{r^{\prime}+r^{\prime \prime}=r \\
l+l^{\prime}=n+1 \\
z_{1}+z_{2}+z_{3}=z}} \frac{1}{(\Lambda+m)^{3+z_{1}}}\left(\sum_{I=0}^{N}\left\|\partial^{z_{2}} \mathcal{D}_{r^{\prime}, 2 l}^{\Lambda ; I}\right\|_{(2 \Lambda, \mu)}\left\|\partial^{z_{3}} \mathcal{L}_{r^{\prime \prime}, 2 l^{\prime}}^{\prime \Lambda ; N-I}\right\|_{(2 \Lambda, \mu)}\right. \\
\left.\left.+\left\|\partial^{z_{2}} \mathcal{D}_{r^{\prime}, 2 l}^{\Lambda ; N}\right\|_{(2 \Lambda, \mu)}\left\|\partial^{z_{3}} \mathcal{D}_{r^{\prime \prime}, 2 l^{\prime}}^{\Lambda}\right\|_{(2 \Lambda, \mu)}\right)\right\}
\end{gathered}
$$

Now we state the factorization

Theorem 4 The difference between two vertex functions, $\mathcal{L}_{r, 2(n, 0)}^{(f) \Lambda}$ of the $\phi$ - $\psi$ theory and $\widetilde{\mathcal{L}^{\prime}}{ }_{r, 2 n}^{\Lambda ; N}$ of the $\phi$ theory, satisfies the bound

$$
\left\|\partial^{z}\left(\mathcal{L}_{r, 2(n, 0)}^{(f) \Lambda}-{\widetilde{\mathcal{L}^{\prime}}}_{r, 2 n}^{\Lambda ; N}\right)\right\|_{(2 \Lambda, \mu)} \leq \begin{cases}\left(\frac{\Lambda+m}{M}\right)^{2 N+2}(\Lambda+m)^{4-2 n-z} \operatorname{Plog}\left(\frac{M}{m}\right), & 0 \leq \Lambda \leq M \\ \left(\frac{\Lambda}{M}\right)^{2 N} \Lambda^{4-2 n-z} \operatorname{Plog}\left(\frac{\Lambda}{m}\right), & M \leq \Lambda \leq \Lambda_{0} .\end{cases}
$$

Proof. Here we use induction on $N, r$ and $n$. The $N=0$ case has been proved already in Theorem 3. Therefore, supposing that Eq. (4.27) is true for $N \leq N^{\prime}-1$ with some $N^{\prime} \geq 1$, we may demonstrate the validity of Eq. (4.27) for $N=N^{\prime}$. Then the rest of the steps are actually very similar to those used in proving Theorem 1 or Theorem 3. If $r=0$ or if $n>n_{0}\left(|r|, N^{\prime}\right)$ for $r$ with $|r|>0$, we know that $\mathcal{L}_{r, 2(n, 0)}^{(f) \Lambda}$ and ${\widetilde{\mathcal{L}^{\prime}}}_{r, 2 n}^{\Lambda ; N^{\prime}}$ (and hence $\mathcal{D}_{r, 2 n}^{\Lambda ; N^{\prime}}$ also) vanish identically and Eq. (4.27) holds trivially for this case. So we prove the validity of Eq. (4.27) for $\partial_{p}^{w} \mathcal{D}_{r, 2 k}^{\Lambda ; N^{\prime}}$ with $|r|=s$ under the assumption that it has been proved for $|r| \leq s-1$, and for $|r|=s$ and $n \geq k+1$. Under this induction hypothesis, Eq. (4.26) is now simplified as

$$
\left\|\partial_{\Lambda} \partial^{z} \mathcal{D}_{r, 2 k}^{\Lambda ; N^{\prime}}\right\|_{(2 \Lambda, \mu)} \leq \begin{cases}\frac{(\Lambda+m)^{5+2 N^{\prime}-2 k-z}}{M^{2 N^{\prime}+2}} \operatorname{Plog}\left(\frac{M}{m}\right), & 0 \leq \Lambda \leq M \\ \frac{\Lambda^{3+2 N^{\prime}-2 k-z}}{M^{2 N^{\prime}}} \operatorname{Plog}\left(\frac{\Lambda}{m}\right), & M \leq \Lambda \leq \Lambda_{0},\end{cases}
$$

where we used Eq. (4.23) in estimating $\left\|\partial^{z_{3}} \mathcal{L}_{r, 2 k}^{\prime \Lambda ; N-I}\right\|_{(2 \Lambda, \mu)}$. Now if $2 k+z>4+2 N^{\prime}$, we go downward from $\Lambda=\Lambda_{0}$ as before and conclude that

$$
\begin{aligned}
\left\|\partial^{z} \mathcal{D}_{r, 2 k}^{\Lambda ; N^{\prime}}\right\|_{(2 \Lambda, \mu)} & \leq \int_{\Lambda}^{\Lambda_{0}} d \lambda\left\|\partial_{\lambda} \partial^{z} \mathcal{D}_{r, 2 k}^{\lambda ; N^{\prime}}\right\|_{(2 \lambda, \mu)} \\
& \leq \begin{cases}\frac{(\Lambda+m)^{6+2 N^{\prime}-2 k-z}}{M^{2 N^{\prime}+2}} \operatorname{Plog}\left(\frac{M}{m}\right), & 0 \leq \Lambda \leq M \\
\frac{\Lambda^{4+2 N^{\prime}-2 k-z}}{M^{2 N^{\prime}}} \operatorname{Plog}\left(\frac{\Lambda}{m}\right), & M \leq \Lambda \leq \Lambda_{0} .\end{cases}
\end{aligned}
$$

(If $2 k+z=5+2 N^{\prime}$, the upper inequality of Eq. (4.29) is obtained using $\partial_{p}^{w} \mathcal{D}_{r, 2 k}^{\Lambda ; N^{\prime}}(0)=0$, as was done in the proof of Theorem 3.) For $2 k+z \leq 4+2 N^{\prime}$, we have

$$
\left|\partial_{p}^{w} \mathcal{D}_{r, 2 k}^{\Lambda ; N^{\prime}}(0)\right| \leq \int_{0}^{\Lambda} d \lambda\left\|\partial_{\lambda} \partial^{z} \mathcal{D}_{r, 2 k}^{\lambda ; N^{\prime}}\right\|_{(2 \lambda, \mu)}
$$


and so Eq. (4.27) follows with the left hand side replaced by $\left|\partial_{p}^{w} \mathcal{D}_{r, 2 k}^{\Lambda ; N^{\prime}}(0)\right|$. Then, again, Taylor's formula (3.22) (now applied to $\mathcal{D}_{r, 2 k}^{\Lambda ; N^{\prime}}$ ) relates the corresponding vertices with those involving twice more differentiation by momenta. Therefore, to complete the proof, induction on $z$ should also be used (for fixed $k$ ). If $z>4+2 N^{\prime}-2 k$, Eq. (4.27) holds as we have just shown above. Now assume that Eq. (4.27) is true for all $z>z_{0}$ for some $z_{0}>0$. Then, finally, Taylor's formula and Eq. (4.30) guarantee the validity of Eq. (4.27) for any $w$ with $|w|=z_{0}$.

If we choose $\Lambda=0$, in Eq. (4.27), it yields the bound for the difference between connected amputated Green functions of the $\phi-\psi$ theory and those of the $\phi$ theory with insertions of appropriate operators defined above. Explicitly, for $\left|p_{i}\right| \leq \mu$,

$$
\left|\partial_{p}^{w}\left(G_{r, 2(n, 0)}^{(f) c}\left(p_{1}, \ldots, p_{2 n-1}\right)-\widetilde{G}_{r, 2 n}^{\prime c ; N_{0}}\left(p_{1}, \ldots, p_{2 n-1}\right)\right)\right| \leq\left(\frac{m}{M}\right)^{2 N_{0}+2} m^{4-2 n-z} \operatorname{Plog}\left(\frac{M}{m}\right),
$$

where $\widetilde{G}_{2 n}^{c ; N_{0}}=\widetilde{\mathcal{L}}_{2 n}^{\Lambda=0 ; N_{0}}=\sum_{N=0}^{N_{0}} G_{2 n}^{c ; N} / N$ !. Thus one may identify the effective theory which is accurate up to order $1 / M^{2 N_{0}}$ as that defined by $\widetilde{L}^{\Lambda_{0} ; N_{0}}=L^{\Lambda_{0}}+\sum_{N=1}^{N_{0}} L^{\Lambda_{0} ; N} / N$ ! and the flow equation (2.10). We will discuss more on this effective action in Sec. 4.3. If $\Lambda$ is larger

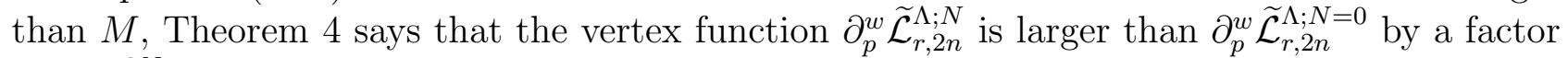
$(\Lambda / M)^{2 N}$. Therefore, in this scale, increasing $N$ (or adding more irrelevant terms, equivalently) makes the vertex functions "unnaturally" large and the theory becomes "less effective". This is because we have imposed unnatural renormalization conditions (4.20) on irrelevant vertices. If we chose natural values as the bare irrelevant couplings, they would give only $O\left(\left(m / \Lambda_{0}\right)^{2 N}\right)$ contributions to Green functions; but, in Eq. (4.20), we imposed the values of order $(m / M)^{2 N}$ to $\widetilde{\mathcal{L}}_{r, 2 n}^{\Lambda ; N}$ as the renormalization conditions, which are natural only if the cutoff is around $M$. This affirms that the effective theory is useful only below the heavy particle mass scale $M$.

The convergence bound as $\Lambda_{0} \rightarrow \infty$ shows a similar behavior. For $\Lambda<M$, the difference converges faster by a factor $\left(\frac{\Lambda+m}{M}\right)^{2 N}$ than the $N=0$ case, while the convergence becomes worse if $\Lambda>M$. We state the result here omitting proof:

$$
\left\|\partial_{\Lambda_{0}} \partial^{z}\left(\mathcal{L}_{r, 2 n}^{(f) \Lambda}-{\widetilde{\mathcal{L}^{\prime}}}_{r, 2 n}^{\Lambda ; N}\right)\right\|_{(2 \Lambda, \mu)} \leq\left(\frac{\Lambda+m}{\Lambda_{0}}\right)^{3}\left(\frac{\Lambda+m}{M}\right)^{2 N}(\Lambda+m)^{3-2 n-z} \operatorname{Plog}\left(\frac{\Lambda_{0}}{m}\right) .
$$

\subsection{Irrelevant operators in the effective Lagrangian}

So far we have shown that, at low energy, connected amputated Green functions of the full theory are reproduced by considering those of the $\phi$ theory plus those with insertion of operators $O_{N}, N=1, \cdots, N_{0}$, defined in Eq. (2.8). Such operator insertions have a simple interpretation if one uses the normal product notation [15, 5] described in the Appendix. Let us begin with $N_{0}=1$. Comparing Eq. (4.20) with Eq. (A.3), we identify $L^{\Lambda_{0} ; 1}$ as

$$
L^{\Lambda_{0} ; 1}=\sum_{2 n+|w|=6} b_{2 n,\{w\}}^{R ; 1}\left[M_{2 n,\{w\}}\right],
$$


where $b_{2 n,\{w\}}^{R ; 1}=\frac{1}{\mathcal{N}_{\{w\}}} \partial_{p}^{w}\left(G_{2(n, 0)}^{(f) c}-G_{2 n}^{c}\right)(0), \mathcal{N}_{\{w\}}$ is a combinatorial factor defined by Eq. (A.3), and $\left[M_{2 n,\{w\}}\right]$ is the normal product of a local vertex

$$
M_{2 n,\{w\}} \equiv \int d^{4} x \partial_{x}^{w_{1}} \phi \cdots \partial_{x}^{w_{2 n-1}} \phi \phi(x)
$$

(see Eq. (A.4)). In terms of the local Euclidean-invariant vertices of dimension six, the above equation can be cast into a more attractive form given in Ref. [13,

$$
\begin{aligned}
L^{\Lambda_{0} ; 1} & =a_{1}^{(6)} \int d^{4} x\left[\phi\left(\partial^{2}\right)^{2} \phi(x)\right]+a_{2}^{(6)} \int d^{4} x\left[\phi^{3} \partial^{2} \phi(x)\right]+a_{3}^{(6)} \int d^{4} x\left[\phi^{6}(x)\right] \\
& \equiv \sum_{i=1}^{3} a_{i}^{(6)}\left[\Omega_{i}^{(6)}\right]
\end{aligned}
$$

with

$$
\begin{aligned}
a_{1}^{(6)} & =\frac{1}{8 \cdot 4 !}\left(\partial^{2}\right)^{2}\left(G_{(2,0)}^{(f) c}-G_{2}^{c}\right)(0), \\
a_{2}^{(6)} & =-\frac{1}{8} \partial^{2}\left(G_{(4,0)}^{(f) c}-G_{4}^{c}\right)(0) \\
a_{3}^{(6)} & =\left(G_{(6,0)}^{(f) c}-G_{6}^{c}\right)(0) .
\end{aligned}
$$

The $1 / M^{2}$-order effects are then described by the insertion of the operator $O_{1}=\sum_{i} a_{i}^{(6)}\left[\Omega_{i}^{(6)}\right]$ :

$$
G_{2(n, 0)}^{(f) c}=G_{2 n}^{c}+\sum_{i=1}^{3} a_{i}^{(6)} G_{2 n}^{c ; 1}\left(\left[\Omega_{i}^{(6)}\right]\right)+O\left(\frac{m^{8-2 n}}{M^{4}} \operatorname{Plog}\left(\frac{M}{m}\right)\right),
$$

where $G_{2 n}^{c ; 1}\left(\left[\Omega_{i}^{(6)}\right]\right)$ denotes the Green function with $\left[\Omega_{i}^{(6)}\right]$ inserted. For general $N_{0}, L^{\Lambda_{0} ; N}(N=$ $\left.1, \ldots, N_{0}\right)$ can be identified as

$$
L^{\Lambda_{0} ; N}=\sum_{2 n+|w|=4+2 N} b_{2 n,\{w\}}^{R ; N}\left[M_{2 n,\{w\}}\right]+L_{C T}^{\Lambda_{0} ; N}
$$

where

$$
b_{2 n,\{w\}}^{R ; N}=\frac{N !}{\mathcal{N}_{\{w\}}} \sum_{2 n+|w|=4+2 N} \partial_{p}^{w}\left(G_{2(n, 0)}^{(f) c}-\widetilde{G}_{2 n}^{c ; N-1}\right)(0),
$$

and $L_{C T}^{\Lambda_{0} ; N}$ denote counterterms which are needed to cancel the new divergences due to the multiple insertion of $L^{\Lambda_{0} ; I}$ 's, $I=1, \ldots, N-1$. (See the discussion of the last paragraph in the Appendix for explicit forms of $L_{C T}^{\Lambda_{0} ; N}$.) Now let $\left[\Omega_{i}^{(4+2 N)}\right]$ 's form a complete set of mutually independent Euclidean-invariant local vertices of dimension $(4+2 N)$ and let $a_{i}^{(4+2 N)}$ 's be appropriate expansion coefficients as in Eq. (4.35), so that we may write

$$
L^{\Lambda_{0} ; N}=\sum_{i} a_{i}^{(4+2 N)}\left[\Omega_{i}^{(4+2 N)}\right]+L_{C T}^{\Lambda_{0} ; N}
$$


Then the effective action $\widetilde{L}^{\Lambda_{0} ; N_{0}}$ may be written as

$$
\begin{aligned}
\widetilde{L}^{\Lambda_{0} ; N_{0}} & =L^{\Lambda_{0}}+\sum_{N=1}^{N_{0}} \sum_{i} \frac{a_{i}^{(4+2 N)}}{N !}\left[\Omega_{i}^{(4+2 N)}\right]+\sum_{N=2}^{N_{0}} \frac{1}{N !} L_{C T}^{\Lambda_{0} ; N} \\
& =\widetilde{L}^{\Lambda_{0} ; N_{0}-1}+\sum_{i} \frac{a_{i}^{\left(4+2 N_{0}\right)}}{N_{0} !}\left[\Omega_{i}^{\left(4+2 N_{0}\right)}\right]+\frac{1}{N_{0} !} L_{C T}^{\Lambda_{0} ; N_{0}}
\end{aligned}
$$

where $a_{i}^{(4+2 N)}$ 's are appropriate linear combinations of $b_{2 n,\{w\}}^{R ; N}$ 's (all of which are $O\left(1 / M^{2 N}\right)$ ). Thus the $1 / M^{2 N}$-order information of the full theory is factorized into the coefficient $a_{i}^{(4+2 N) \text {, }}$ of local vertices of dimension $(4+2 N)$. Given this bare Lagrangian, the flow equation guarantees that all Green functions are finite and accurate up to order $1 / M^{2 N}$.

The interpretation of this result is clear from the viewpoint of the renormalization group flow in the infinite dimensional space of possible Lagrangians. As we reduce the cutoff, the bare Lagrangian of the full theory, which is specified by the boundary conditions (3.11) and (3.12), flows down to a submanifold parametrized by relevant couplings only. It has both heavy and light operators. Now the flow may be projected onto the submanifold of operators consisting of the light field only. Then finding a local low-energy effective field theory of light particles is equivalent to finding a renormalization group flow with a few (relevant or irrelevant) local operators which can best approximate the projected flow of the full theory. To the zeroth order in $1 / M^{2}$, it is done with relevant terms only by choosing the same renormalization conditions as those of the full theory. The deviation from the full-theory flow is corrected by reading off values of the remaining irrelevant coordinates at the $\Lambda=0$ point. At first, components of dimension six operators may be read, which tell us the effects of order $1 / M^{2}$; the flow of the full theory is now approximated to the order $1 / M^{2}$ at $\Lambda<M$. If one wants to increase the accuracy, it is necessary to read more and more irrelevant coordinates (at $\Lambda=0$ ) of the projected flow of the full theory and modify the effective-theory flow with the corresponding flow equation given in Eq. (2.10). Our equation (2.10) automatically takes care of possible divergences due to the unnaturally large irrelevant components (see the discussion of Sec. 1) in such a way that the bare Lagrangian may contain only a finite number of irrelevant terms.

\section{CONCLUSIONS}

In the context of a simple scalar field theory, we have demonstrated that, at low energy, virtual heavy particle effects on light-particle Green functions are completely factorized via effective local vertices to any desired order. For this, we have used the powerful flow equation approach which can be regarded as a differential version of Wilson's renormalization group transformation. Remarkably, the method which has been used previously to prove renormalizability is found to have an immediate generalization to this problem. In applying this method, we have seen that irrelevant terms in the Wilsonian effective Lagrangian (which has a finite UV cutoff $\Lambda=M$, a characteristic scale representing heavy-particle thresholds) are replaceable by the corresponding higher dimensional composite operators plus counterterms for their 
products in the conventional approach (where UV cutoff is supposed to go to infinity). The latter can be dealt with with the help of the normal product concept. Once this fact is noticed, factorization is straightforward: all $1 / M^{2 N}$-order effects can be isolated in terms of local vertices involving operators of dimension $(4+2 N)$. Thereby we arrive at a local effective field theory which describes low-energy light-particle physics accurately up to any desired order in $1 / M^{2}$ with appropriate calculation rules for irrelevant (nonrenormalizable) vertices. Since the arguments here are essentially dimensional, it should not be difficult to generalize them to different field theories such as gauge theories or theories with spontaneous symmetry breaking. As for these models, we note that the flow equation approach has already been used to discuss renormalizability and related problems [3, 5, 6].

In this paper we limited our attention to vertex functions at momentum range $\mu=O(m)$. As one increases $\mu$ to sufficiently high energy scale, the $(\mu / m)$-dependence we neglected will become important. Keeping such $\mu$-dependence and establishing bounds more carefully, one may study the high-momentum behavior of Green functions (even in the exceptional momentum region) within the effective field theory context. In this regard, we note that the infrared behavior of massless scalar theory has been analyzed by using flow equations recently [5, 6]. Another related point is the following. Effective theory is supposed to be useful mainly at low energy. As we increase energy, we need in fact more and more irrelevant terms to maintain the same level of accuracy. Conversely, with a few irrelevant terms added to the Lagrangian, we may extend the energy range where it remains effective. Then it seems worth investigating quantitatively to what energy scale the given effective theory may be used to reproduce the full-theory results with reasonable accuracy.

\section{NOTE ADDED}

Completing the main parts of this paper, we became aware of the very recent paper by Girardello and Zaffaroni [20] which has a partial overlap with the present paper. They also discussed decoupling and factorization to order $1 / M^{2}$ using flow equations. However, we believe that our treatment is physically more transparent and systematic. For example, they did not discuss the normal product description nor higher order generalization; this does not look trivial

in their method for, in their approach to these issues, nonnegligible parts of heavy-field modes might remain unintegrated even at scales much less than the heavy field mass $M$.

\section{ACKNOWLEDGMENTS}

It is a great pleasure to thank Professor Choonkyu Lee for suggesting this problem and carefully reading the manuscript. This work was supported in part by the Korea Science and Engineering Foundation (through Center for Theoretical Physics, SNU) and by the Basic Science Research Institute Program (Project No. BSRI-94-2418), Ministry of Education, Korea. 


\section{APPENDIX}

Here we briefly discuss the renormalization of composite operators and their products «, 5], within the $\phi^{4}$-theory defined by $L^{\Lambda_{0}}$ in Eq. (4.1). We restrict our discussions to those needed in the main part of this paper. The operator $L^{\Lambda_{0} ; N}$ 's of Sec. 2 are assumed to be of local, even polynomials in $\phi$ and its derivatives of dim. $\leq 2 D_{N}$,

$$
L^{\Lambda_{0} ; N}=\sum_{n=1}^{D_{N}} \sum_{2 n+|w| \leq 2 D_{N}} b_{2 n,\{w\}}^{N} M_{2 n,\{w\}}
$$

where $b_{2 n,\{w\}}^{N}$ is a formal power series in $g_{1}, b_{2 n,\{w\}}^{N}=\sum_{r=0}^{\infty} g_{1}^{r} b_{r ; 2 n,\{w\}}^{N}$, which is to be determined later uniquely when we impose renormalization conditions. [ $M_{2 n,\{w\}}$ is defined in Eq. (4.34).] Throughout this Appendix, the dimension $D_{N}$ is restricted to satisfy the condition $\left(2 D_{N}-4\right) \geq$ $\sum_{i=1}^{k} N_{i}\left(2 D_{i}-4\right)$, where $\left.D_{i} \equiv D_{N}\right|_{N_{j}=\delta_{i j}}, N=\left(N_{1}, \ldots, N_{k}\right)$. We follow the now familiar procedure. $\mathcal{L}_{r, 2 n}^{\Lambda ; N}$ s are defined by Eq. (4.17) and have similar properties except for the Euclidean invariance . They satisfy the flow equation (4.18). Boundary conditions are given similarly:

$$
\begin{array}{ccc}
\Lambda=\Lambda_{0}: & \partial_{p}^{w} \mathcal{L}_{r, 2 n}^{\Lambda_{0} ; N}=0, & \text { for } 2 n+|w|>2 D_{N}, \\
\Lambda=0: & \partial_{p}^{w} \mathcal{L}_{r, 2 n}^{\Lambda=0 ; N}(0)=b_{r ; 2 n,\{w\}}^{R ; N} \mathcal{N}_{\{w\}}, & \text { for } 2 n+|w| \leq 2 D_{N},
\end{array}
$$

where $\mathcal{N}_{\{w\}}$ is a combinatorial factor defined by $\left.\partial_{p}^{w^{\prime}}\left[\left(i p_{1}\right)^{w_{1}} \cdots\left(i p_{2 n-1}\right)^{w_{2 n-1}}\right]_{s y m m}\right|_{p=0}=\delta_{\{w\}\left\{w^{\prime}\right\}} \mathcal{N}_{\{w\}}$, and $b_{r ; 2 n,\{w\}}^{R ; N}$ 's are finite and $\Lambda_{0}$-independent constants. [Here it is to be noted that $b_{2 n,\{w\}}^{N}$ and $b_{2 n,\{w\}}^{R ; N}$ are in general not the same; this is the usual operator mixing [4, 用.] With these one can easily prove the perturbative renormalizability of Green functions with the insertion of operator $O_{N}$ (which is defined in Eq. (2.8)); the proof is just a simpler version of that in Theorem 4 .

We now discuss Zimmermann's normal product [15], following Ref. [5]| W. We begin with a monomial for the case of $k=N=1$ with $D_{1} \equiv D$. Normal product $\left[M_{2 n^{\prime},\left\{w^{\prime}\right\}}\right]_{2 D}$ of dimension $2 D\left(\geq 2 n^{\prime}+\left|w^{\prime}\right|\right)$ is defined by requiring that the vertices with one insertion of $O_{1}=\left[M_{2 n^{\prime},\left\{w^{\prime}\right\}}\right]_{2 D}$, which are denoted as $\mathcal{L}_{r, 2 n}^{\Lambda ; 1}\left(\left[M_{2 n^{\prime},\left\{w^{\prime}\right\}}\right]_{2 D}\right)$, obey the renormalization condition (A.3) with

$$
b_{r ; 2 n,\{w\}}^{R ; 1}=\delta_{n n^{\prime}} \delta_{\{w\}\left\{w^{\prime}\right\}} \delta_{r 0} .
$$

Since the flow equation is linear, for general boundary condition (A.3) we may write $L^{\Lambda_{0} ; 1}$ as

$$
L^{\Lambda_{0} ; 1}=\sum_{2 n+\{w\} \leq 2 D} b_{2 n,\{w\}}^{R ; 1}\left[M_{2 n,\{w\}}\right]_{2 D} \equiv\left[\sum_{2 n+\{w\} \leq 2 D} b_{2 n,\{w\}}^{R ; 1} M_{2 n,\{w\}}\right]_{2 D} .
$$

\footnotetext{
${ }^{5}$ Since the flow equation respects $O(4)$ Euclidean symmetry, one may well restrict the consideration to Euclidean-invariant operators and then $\mathcal{L}_{r, 2 n}^{\Lambda ; N}$ will also be Euclidean invariant.

${ }^{6}$ Here we are discussing only space-integrated operators, or vertices. Generalization to local operators, e.g. $\left[M_{2 n,\{w\}}(x)\right]$, are straightforward and is described in Ref. [5] to the order $k=2$ and $|N|=2$.
} 
When the dimension of the operator is equal to $2 D$, we simply write $[\cdots]_{2 D}=[\cdots]$; for example, $\left[M_{2 n,\{w\}}\right]_{2 D}=\left[M_{2 n,\{w\}}\right]$ if $2 D=2 n+|w|$. Therefore, if we choose $b_{2 n,\{w\}}^{R ; 1}=0$ for $2 n+|w| \neq 2 D$, we may omit the subscript $D$ in Eq. (A.5). This is the case of Sec. 4 (see Eq. (4.33)).

The normal product of several operators (assuming $k>1$ ) are defined inductively as follows. Let us write $O_{N}=L^{\Lambda_{0} ; N}=\left[B_{i}\right]_{2 D_{i}}$ for $N=\left(N_{1}, \ldots, N_{k}\right)$ with $N_{j}=\delta_{i j}$ (i.e., $\left.|N|=1\right)$. Suppose that $\left[\left[B_{i_{1}}\right]_{2 D_{i_{1}}} \cdots\left[B_{i_{k-1}}\right]_{2 D_{i_{k-1}}}\right]_{2 D}$ 's have been defined, where $(2 D-4) \geq \sum_{l=1}^{k-1}\left(2 D_{i_{l}}-4\right)$, $1 \leq i_{l} \leq k$. Then we define the normal product $\left[\left[B_{1}\right]_{2 D_{1}} \cdots\left[B_{k}\right]_{2 D_{k}}\right]_{2 D_{N}}$ as $O_{(1, \ldots, 1)}$ which flows down through Eq. (2.10) with the simplest boundary condition

$$
\partial_{p}^{w} \mathcal{L}_{r, 2 n}^{\Lambda=0 ;(1, \ldots, 1)}(0)=b_{r ; 2 n,\{w\}}^{R ;(1, \ldots, 1)} \mathcal{N}_{\{w\}}=0, \quad 2 n+|w| \leq 2 D_{N} .
$$

If $\left(2 D_{N}-4\right)=\sum_{i}\left(2 D_{i}-4\right)$, we write $[\cdots]_{2 D_{N}} \equiv[\cdots]$ as above. Let us illustrate this definition for $k=2$ and $k=3$ cases. If $k=2$, [[B $\left.]_{2 D_{1}}\left[B_{2}\right]_{2 D_{2}}\right]_{2 D_{(1,1)}}$ is defined as

$$
\left[\left[B_{1}\right]_{2 D_{1}}\left[B_{2}\right]_{2 D_{2}}\right]_{2 D_{(1,1)}} \equiv O_{(1,1)}=\left[B_{1}\right]_{2 D_{1}}\left[B_{2}\right]_{2 D_{2}}+L^{\Lambda_{0} ;(1,1)}
$$

where the flow of $L^{\Lambda_{0} ;(1,1)}$ is governed by Eq. (2.10) together with the boundary condition (A.6). Therefore, $L^{\Lambda_{0} ;(1,1)}$ is interpreted as the counterterm needed to cancel divergences appearing in Green functions with the insertion of the product operator $\left[B_{1}\right]_{2 D_{1}}\left[B_{2}\right]_{2 D_{2}}$. Similarly, if $k=3$, from the expression for $O_{(1,1,1)}$ given in Eq. (2.9) we have

$$
\begin{aligned}
{\left[\left[B_{1}\right]_{2 D_{1}}\left[B_{2}\right]_{2 D_{2}}\left[B_{3}\right]_{2 D_{3}}\right]_{2 D_{(1,1,1)}} \equiv } & O_{(1,1,1)} \\
= & {\left[B_{1}\right]_{2 D_{1}}\left[B_{2}\right]_{2 D_{2}}\left[B_{3}\right]_{2 D_{3}}+\left[B_{1}\right]_{2 D_{1}} L^{\Lambda_{0} ;(0,1,1)} } \\
& +\left[B_{2}\right]_{2 D_{2}} L^{\Lambda_{0} ;(1,0,1)}+\left[B_{3}\right]_{2 D_{3}} L^{\Lambda_{0} ;(1,1,0)}+L^{\Lambda_{0} ;(1,1,1)}
\end{aligned}
$$

where $L^{\Lambda_{0} ;(1,1,0)}$ etc. are given by Eq. (A.7), and additional divergences due to multiple insertions are cancelled by $L^{\Lambda_{0} ;(1,1,1)}$ which obeys the flow equation $(2.10)$ with $N=(1,1,1)$, with the boundary condition (A.6). In this way, $\left[\left[B_{1}\right]_{2 D_{1}} \cdots\left[B_{k}\right]_{2 D_{k}}\right]_{2 D_{N}}$ is defined for general $k$, i.e., $L^{\Lambda_{0} ;(1, \ldots, 1)}$ serves as the "counterterm" for new divergences appearing in Green functions due to the new products of lower order operators and $\left[\left[B_{1}\right]_{2 D_{1}} \cdots\left[B_{k}\right]_{2 D_{k}}\right]_{2 D_{N}}$ is interpreted as the resulting "subtracted" finite part. Since this definition satisfies multilinearity, which can be proved by use of induction on $k$ while taking into account Eqs. (2.9) and (2.10), and boundary conditions, we can freely put the coefficients multiplying operators inside or outside the brackets.

For the general boundary conditions (A.3) where $b_{2 n,\{w\}}^{R ; N}$ need not be zero, we recall the property of the flow equation (2.10) mentioned at the end of Sec. 2. The general solution of the flow equation is represented as a sum of a particular solution and a solution of the homogeneous equation which is identical to the flow equation with $|N|=1$, i.e.,

$$
L^{\Lambda ; N}=L^{\Lambda ; 1}\left(\left[B_{N}\right]_{2 D_{N}}\right)+L_{C T}^{\Lambda ; N}
$$

Here we may absorb all the nonzero boundary values into $L^{\Lambda_{0} ; 1}\left(\left[B_{N}\right]_{2 D_{N}}\right)$ choosing

$$
\left[B_{N}\right]_{2 D_{N}}=\sum_{2 n+|w| \leq 2 D_{N}} b_{2 n,\{w\}}^{R ; N}\left[M_{2 n,\{w\}}\right]_{2 D_{N}}
$$


and then $L_{C T}^{\Lambda ; N}$ satisfies the simplest boundary condition (A.6). (The subscript $C T$ is attached to indicate its counterterm nature as discussed above.) In other words, $b_{2 n,\{w\}}^{R ; N} \neq 0$ corresponds to adding new composite operator $\left[B_{N}\right]_{2 D_{N}}$ to $L^{\Lambda_{0} ; N}$ besides the counterterms needed just to cancel divergences associated with the multiple insertion of operators already included. For example,if $k=2$, we may extend Eq. (A.7) to the general case,

$$
\begin{aligned}
L^{\Lambda_{0} ;(1,1)} & =L_{C T}^{\Lambda_{0} ;(1,1)}+\left[B_{(1,1)}\right]_{2 D_{(1,1)}} \\
& =\left[\left[B_{1}\right]_{2 D_{1}}\left[B_{2}\right]_{2 D_{2}}\right]_{2 D_{(1,1)}}-\left[B_{1}\right]_{2 D_{1}}\left[B_{2}\right]_{2 D_{2}}+\sum_{2 n+|w| \leq 2 D_{(1,1)}} b_{2 n,\{w\}}^{R ;(1,1)}\left[M_{2 n,\{w\}}\right]_{2 D_{(1,1)}} .
\end{aligned}
$$

This is the case we encountered in Sec. 4.

\section{REFERENCES}

1. K. G. Wilson, Phys. Rev. B 4 (1971), 3174, 3184.

2. J. Polchinski, Nucl. Phys. B 231 (1984), 269.

3. B. J. Warr, Ann. Phys. (N. Y.) 183 (1988), 1, 89.

4. J. Hughes and J. Liu, Nucl. Phys. B 161 (1988), 183.

5. G. Keller, C. Kopper and M. Salmhofer, Helv. Phys. Acta 65 (1992), 32; G. Keller and C. Kopper, Phys. Lett. B 273 (1991), 323; Comm. Math. Phys. 148 (1992), 445; 153 (1993), 245; Comm. Math. Phys. 161 (1994), 515.

6. M. Bonini, M. D’Attanosio and G. Marchesini, Nucl. Phys. B 409 (1993),441; 418 (1994),81.

7. C. Wetterich, Phys. Lett. B 301 (1993), 90 and references therein.

8. U. Ellwanger, Z. Phys. C 62 (1994), 503.

9. T. R. Morris, Int. J. Mod. Phys. A 9 (1994,2411.

10. R. D. Ball and R. S. Thorne, CERN-TH.7067/93, OUTP-93-23P.

11. T. Appelquist and J. Carazzone, Phys. Rev. D 11 (1975), 2856.

12. S. Weinberg, Phys. Lett. B 91 (1980), 51.

13. C. Lee, Nucl. Phys. B 161 (1979), 171.

14. Y. Kazama and Y. P. Yao, Phys. Rev. D 21 (1980), 1116, 1138.

15. W. Zimmermann, in "Lectures on Elmentary Particles and Fields", (S. Deser, M. Grisaru and H. Pendleton, Eds) MIT Press, Cambridge, MA, 1970. 
16. K. Symanzik, Nucl. Phys. B 226 (1983), 187, 205.

17. C. Wieczerkowski, Comm. Math. Phys. 120 (1988), 148; G. Keller, Helv. Phys. Acta 66 (1993), 453.

18. See for example J. C. Collins, "Renormalization", Cambridge University press, 1984.

19. See for example B. Ovrut and H. Schnitzer, Phys. Rev. D 22 (1980), 3369; D 22 (1980), 2518; H. Georgi, Nucl. Phys. (Proc. Suppl.) 29B,C (1992), 1.

20. L. Girardello and A. Zaffaroni, Nucl. Phys. B 424 (1994), 219. 


\section{Figure caption}

Fig. 1. Graphical representation of the flow equation (3.10). Thin (thick) lines represents light (heavy) field, and numerical factors in Eq. (3.10) are ignored here. 
This figure "fig1-1.png" is available in "png" format from: http://arxiv.org/ps/hep-th/9402117v3 


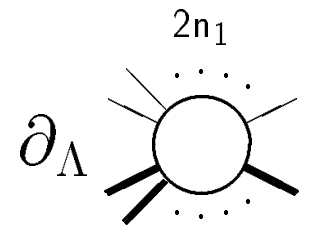

$2 n_{2}$

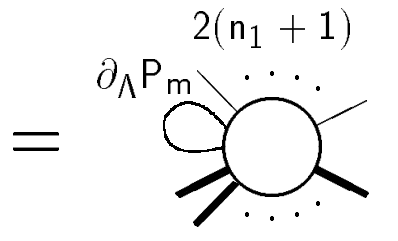

$2 n_{2}$
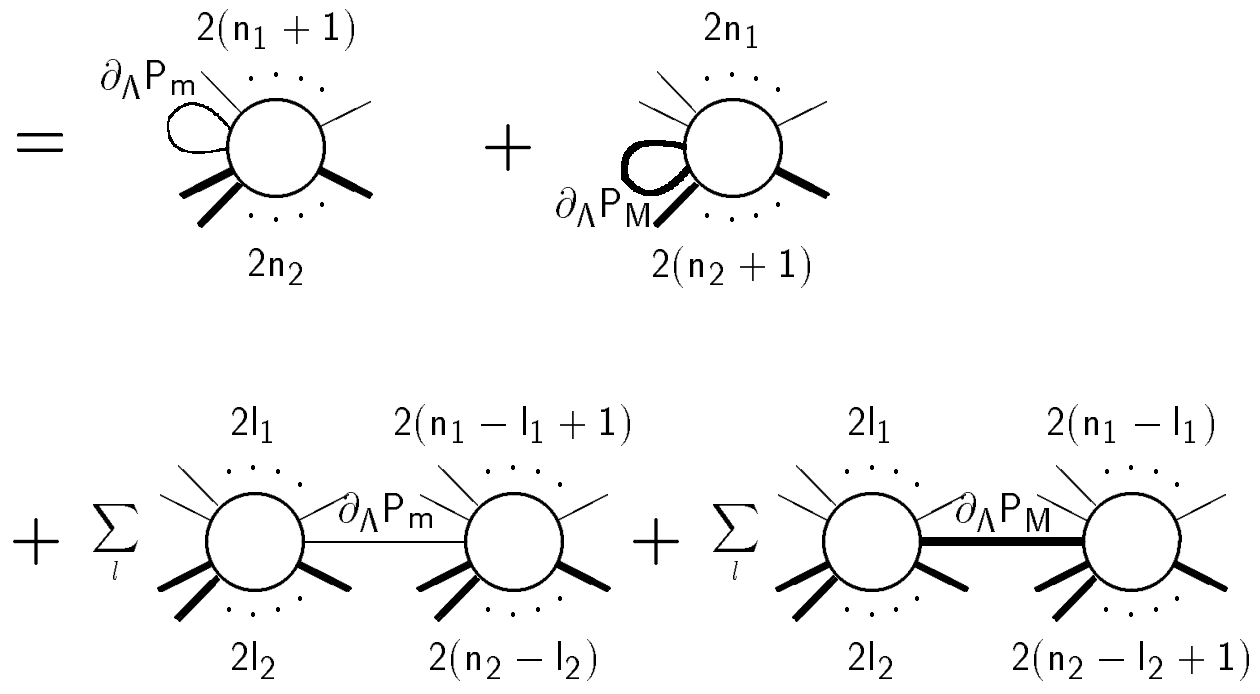